\title{
U-Pb zircon geochronology and petrology of granitoids from Mitwaba (Katanga, Congo): implications for the evolution of the Mesoproterozoic Kibaran belt
}

\author{
J. Kokonyangi ${ }^{\mathrm{a}, *}$, R. Armstrong ${ }^{\mathrm{b}}$, A.B. Kampunzu ${ }^{\mathrm{c}}$, M. Yoshida ${ }^{\mathrm{d}}$, T. Okudaira $^{\mathrm{a}}$ \\ a Department of Geoscience, Faculty of Science, Osaka City University, Sugimoto 3-3-138 Sumiyoshi-ku, Osaka 558-8585, Japan \\ ${ }^{\mathrm{b}}$ Research School of Earth Sciences, The Australian National University, Canberra, ACT 0200, Australia \\ ${ }^{c}$ University of Botswana, Private Bag 0022, Gaborone, Botswana \\ ${ }^{\mathrm{d}}$ Gondwana Institute of Geology and Environment, 147-2 Hashiramoto, Hashimoto 648-0091, Japan
}

Received 17 April 2002; accepted 12 February 2004

\begin{abstract}
In Katanga (Congo), Mesoproterozoic Kibaran granitoids were previously divided into: (1) type A or $\mathrm{G}_{1}$ granitoids (1370 \pm $25 \mathrm{Ma}$ ); (2) type $\mathrm{B}$ or $\mathrm{G}_{2}$ granitoids (1308 $\pm 25 \mathrm{Ma}$ ); (3) type $\mathrm{C}$ or $\mathrm{G}_{3}$ granitoids (1094 $\left.\pm 50 \mathrm{Ma}\right)$; and (4) type $\mathrm{D}$ or $\mathrm{G}_{4}$ tin granites (ca. $977 \pm 18 \mathrm{Ma}$ ). Our field investigations indicate that igneous bodies previously described as $\mathrm{G}_{1}(A), \mathrm{G}_{2}(B)$ and $\mathrm{G}_{3}$ (C) granitoids in the Mitwaba region were emplaced during the same early Kibaran compressional orogenic event $\left(\mathrm{D}_{1}\right)$. The heterogeneous distribution of strain during $\mathrm{D}_{1}$ produced orthogneissic rocks along high strain zones versus slightly to unfoliated granitoids in low strain zones. New SHRIMP U-Pb zircon analyses yield the following igneous crystallisation ages for Mitwaba $\mathrm{G}_{1}-\mathrm{G}_{3}$ granitoids: Kisele monzogranite gneiss $1386.3 \pm 8.1 \mathrm{Ma}$, Kabonvia granodioritic augen gneiss $1385.5 \pm 7.4 \mathrm{Ma}$, Nyangwa monzogranite $1383.3 \pm 4.8 \mathrm{Ma}$, Kungwe-Kalumengongo monzogranite $1377 \pm 10 \mathrm{Ma}$ and Fwifwi foliated leucomonzogranite $1372 \pm 9.6 \mathrm{Ma}$. All these granitoids were emplaced over a short period of time and could be coeval at $1381 \pm 8 \mathrm{Ma}$. This age dates the early Kibaran orogenic event $\left(D_{1}\right)$ in the Mitwaba region. Metamorphic zircons from the strongly deformed Kisele monzogranite gneiss yield an age of $1079 \pm 14 \mathrm{Ma}$, which possibly dates the $\mathrm{D}_{2}$ orogenic event in the Mitwaba area. The tin granites are younger and were emplaced at $\sim 1.0 \mathrm{Ga}$.

The Mitwaba granitoids are two-micas and ilmenite-bearing strongly peraluminous S-type granitoids. The rare-earth elements (REE) patterns show a strong Eu negative anomaly, an enrichment and fractionation of light REE (LREE) and slightly fractionated to almost flat Heavy REE (HREE) patterns. Primordial-mantle normalized spidergrams are characterized by negative anomalies of $\mathrm{Ti}, \mathrm{Sr}, \mathrm{Nb}$ and subsidiary $\mathrm{Ba}$. The emplacement of arc mafic magmas into the crust triggered the dehydration melting, at high temperatures, of a highly fertile and heterogenous metasedimentary source material producing a felsic melt which was mixed with the arc mafic magmas in variable proportions to produce these granitoids. The petrogenesis of these rocks and similar coeval occurrences defining a ca. $3000 \mathrm{~km}$ granitoid belt stretching from SE Congo to NE Congo, Burundi, Rwanda and Uganda indicates similar processes throughout the Kibaran orogenic system in central and eastern Africa.
\end{abstract}

(c) 2004 Elsevier B.V. All rights reserved.

Keywords: Mesoproterozoic; S-type granites; Subduction; Kibaran belt; Congo

\footnotetext{
* Corresponding author. Fax: +81-6-6605-2522.

E-mail address: joseph@sci.osaka-cu.ac.jp (J. Kokonyangi).
} 


\section{Introduction}

The Kibaran belt represents one of the few well-preserved Mesoproterozoic belts in the world (Kampunzu, 2001). The orogenic history of this belt is poorly constrained in the Democratic Republic of Congo (hereafter Congo), because of the lack of modern structural, petrological, geochemical and precise geochronological data. The type area of the Kibaran orogenic system of Africa, known as the Kibaran belt in Katanga, Congo (Cahen et al., 1984 and references therein), runs over $700 \mathrm{~km}$ with an average width of $\sim 300 \mathrm{~km}$. The total Kibaran orogenic system of central and southern Africa is more than $3000 \mathrm{~km}$ long and hosts large volume of granitoids previously suggested to be emplaced along a plate convergent margin (Kampunzu et al., 1986) or to mark an extensional rift setting (e.g. Klerkx et al., 1987). To evaluate these conflicting interpretations, we conducted multidisciplinary studies of granitoids in the Mitwaba region of SE Congo. This paper provides: (1) the first precise $\mathrm{U}-\mathrm{Pb}$ zircon crystallisation ages of a representative set of intrusive bodies allowing constraints to be placed on the timing of deformation and emplacement of granitoid batholiths in the Kibaran type-area; (2) petrological data constraining the genesis and tectonic setting during the emplacement of granitoids.

\section{Regional geology and previous geochronology}

The Kibaran orogenic system of Africa includes the following belts (Fig. 1): Kibaran (SE Congo), Burundian (E Congo, Rwanda, and Burundi), KaragweAnkolean (SE Uganda and NW Tanzania), Luhule Mobissio and Maniema (NE Congo), Irumides (Zambia),

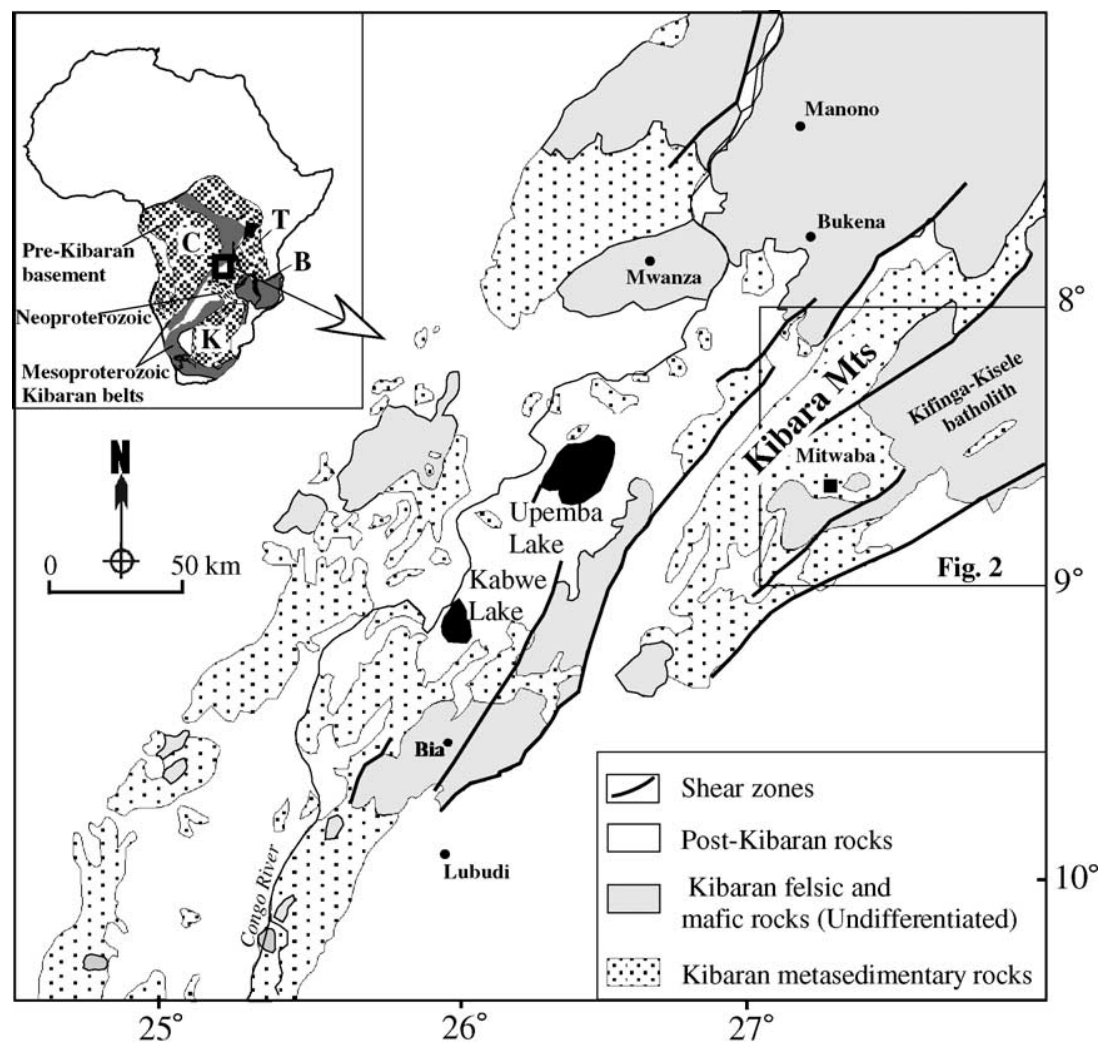

Fig. 1. Sketch map of the Kibaride belt in Central Katanga (Congo) showing the distribution of Mesoproterozoic granitoids, mafic igneous and metasedimentary rocks. The upper inset shows the Mesoproterozoic Kibaran belts in southern Africa and the pre-Kibaran basements. C, Congo, T, Tanzania, B, Bangweulu and K, Kalahari cratons. 
Namaqua-Sinclair-Rehoboth (Namibia), NamaquaNatal (South Africa) and Lurio (MozambiqueMalawi). The Kibaran belt defines a NE-trending belt exposed in SE Congo (Fig. 1). It is bounded by the Archaean/Paleoproterozoic Congo craton to the northwest. To the southeast, it is in contact with a mosaic of older crustal provinces amalgamated before the Kibaran orogeny, including the Palaeoproterozoic Bangweulu Block (Kapenda et al., 1998) and the Archaean Tanzania craton (Fig. 1). There are no geological similarities between the rock assemblages of the Bangweulu block/Tanzania craton and those of the Congo craton on the two margins of the Kibaran belt.

The Kibaran Supergroup is a thick supracrustal sedimentary succession $(>10,000 \mathrm{~m}$; Cahen et al., 1984), consisting mainly of metamorphosed pelites, siltstones, sandstones and conglomerates, with minor carbonate rocks. Large felsic and mafic igneous bodies intrude these metasedimentary rocks (e.g. Cahen, 1954; Kampunzu et al., 1986). Kibaran metasedimentary and igneous rocks were affected by two main compressional events (e.g. Kampunzu et al., 1986, 1998; Rumvegeri, 1991). The timing of these contractional events was inferred to be in the 1400-1000 Ma bracket (e.g. Cahen et al., 1984). Kampunzu et al. (1998) consider that the first event marks the development of an active continental margin, between 1.4 and $1.25 \mathrm{Ga}$. The second event is taken to mark a continental collision $(1.25-1.0 \mathrm{Ga})$, with production of late- to post-kinematic "fertile" granites.

The paroxysmal Kibaran compressional deformation $\left(\mathrm{D}_{2}\right)$ generated NE-trending and NW-verging isoclinal folds and thrusts, defining the main physiographic feature of the belt over more than $3000 \mathrm{~km}$, from Katanga in SE Congo to Uganda (Cahen et al., 1984; Kampunzu et al., 1986; Rumvegeri, 1991). Early Kibaran granitoids and related orthogneisses were emplaced during a protracted period between 1.4 and $1.25 \mathrm{Ga}$ (e.g. Cahen et al., 1984; Kampunzu et al., 1998). This suggestion was mainly based on: (1) lowprecision whole-rock $\mathrm{Rb}-\mathrm{Sr}$ isochron and $\mathrm{K}-\mathrm{Ar}$ dates (e.g. Cahen et al., 1984); (2) conventional U-Pb bulk zircon dates (e.g. Ledent, 1979).

In the Kibaran belt, four major granitoid groups were defined by previous workers (e.g. Boutakoff, 1939; Cahen et al., 1984): (1) type $A$ or $\mathrm{G}_{1}$ granitoids yielded a $\mathrm{Rb}-\mathrm{Sr}$ whole-rock isochron of $1331 \pm 25 \mathrm{Ma}$ in the Mitwaba area. For the whole Kibaran orogenic system, Cahen et al. (1984) suggested that an average age of $1370 \pm 25 \mathrm{Ma}$ could be considered for this group; (2) type $\mathrm{B}$ or $\mathrm{G}_{2}$ granitoids for which an average age of $1308 \pm 25 \mathrm{Ma}$ was proposed; (3) type $\mathrm{C}$ or $\mathrm{G}_{3}$ granitoids inferred to be emplaced at around $1094 \pm$ $50 \mathrm{Ma}$; and (4) type $\mathrm{D}$ or $\mathrm{G}_{4}$ tin granites emplaced at ca. $977 \pm 18 \mathrm{Ma}$. This subdivision was followed, with minor modifications, by several authors (e.g. Klerkx et al., 1987; Fernandez-Alonso et al., 1986; Tack et al., 1994), despite the fact that it was poorly constrained by field data and based on low-precision geochronological data. Kampunzu et al. (1986), using a large amount of structural and petrofabric data, suggested that $G_{1}, G_{2}$ and $G_{3}$ Kibaran granitoids were part of the same syn- $\mathrm{D}_{1}$ igneous event. According to these authors, the stronger versus weaker solid-state fabric recorded in $G_{1}$ versus $G_{3}$ (respectively) result from the heterogeneous distribution of strain within the belt instead of reflecting any absolute age difference. The new geochronological data presented in this paper allow us to constrain these conflicting interpretations.

\section{Geology of the Mitwaba area}

Two informal lithostratigraphic units separated by a disconformity were defined within the Kibaran supracrustal metasedimentary sequences in the Mitwaba area (Fig. 2): the older Mitwaba group and the younger Mulumbi group (Kokonyangi et al., 2001). The Mitwaba group includes metapelitic rocks, with minor intercalations of quartzite. This supracrustal metasedimentary assemblage is intruded by gabbros and granitoids. Two main mafic igneous bodies occur within the Mitwaba group (Fig. 2): (1) the Lwabwe metagabbro, enveloping the western margin of the Kifinga-Kisele granitic batholith, intrudes (cf. chilled margin) greenschist facies Mitwaba group metasedimentary rocks; and (2) the Kidilo amphibolite intruding the Mitwaba group supracrustal assemblage to the southeast of this batholith. The contact between the metagabbro and the granitoids is locally well exposed (e.g. near Komeshia), a sharp intrusive contact is observed and the granite contains metagabbro enclaves. The metagabbro is affected by contact metamorphism (static biotite-garnet-hornblende porphyroblasts) and hydrothermal silicification near the granite body (Kokonyangi et al., 2001). 


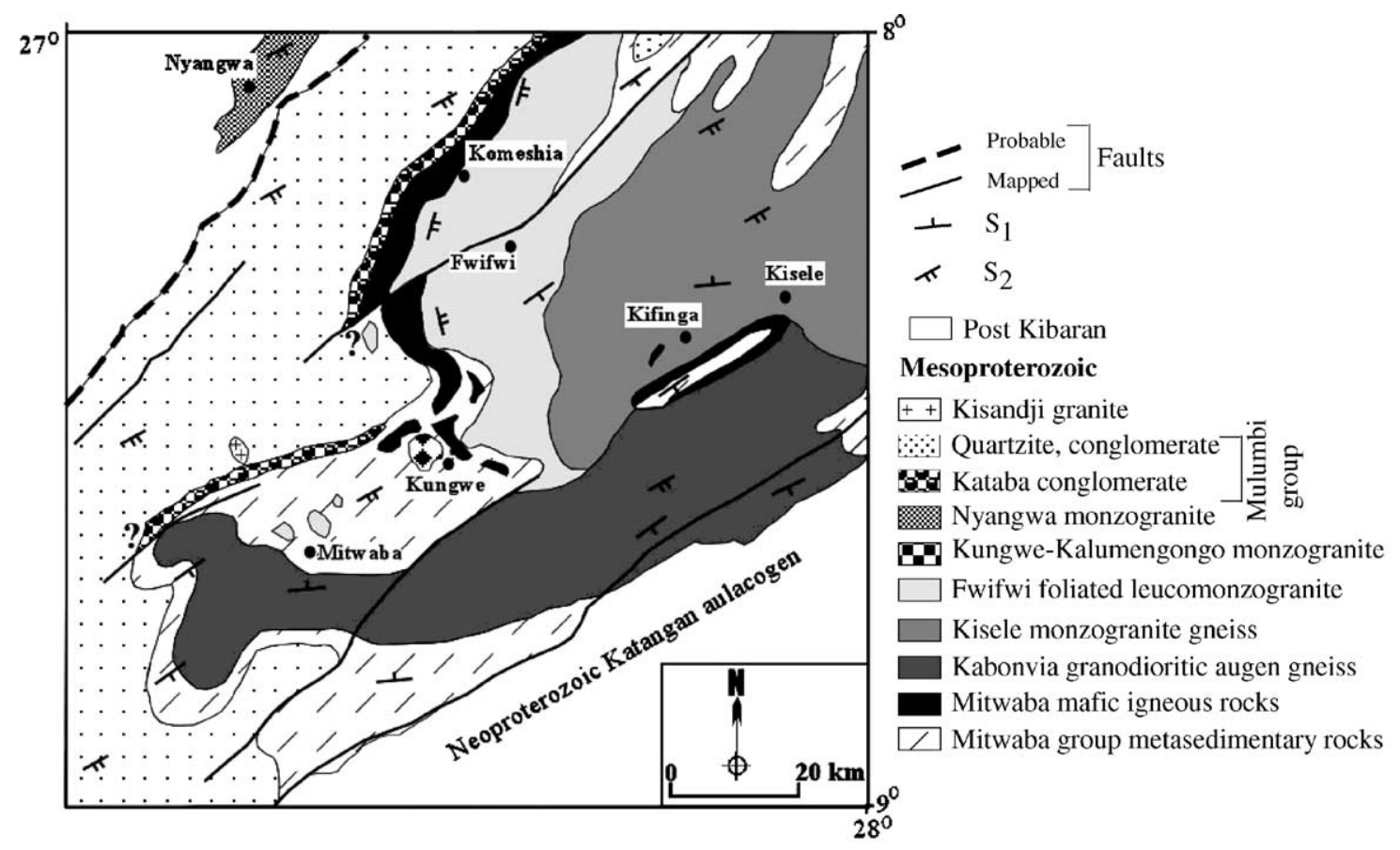

Fig. 2. Geological map of the Mitwaba degree-sheet showing the studied granitoids and associated mafic igneous and sedimentary rocks.

Geochemical characteristics of the Mitwaba mafic igneous rocks indicate a continental arc signature and these mafic rocks yielded $\mathrm{U}-\mathrm{Pb}$ single zircon igneous crystallisation ages between 1.4 and $1.38 \mathrm{Ga}$ (Kokonyangi, unpublished data), supporting field studies indicating that they are closely related to the emplacement of the granitoids.

Two main deformation events $\left(\mathrm{D}_{1}\right.$ and $\left.\mathrm{D}_{2}\right)$ affected the Mitwaba group supracrustal metasedimentary units and related igneous rocks. The oldest contractional deformation $\left(D_{1}\right)$ is characterized by ENE-trending penetrative structures, asymmetric mesoscopic folds $\left(\mathrm{F}_{1}\right)$ with an axial planar foliation $\left(\mathrm{S}_{1}\right)$ steeply dipping $\left(50-60^{\circ}\right)$ to the NW or SE. This fabric is well developed in upper amphibolite facies rocks of the Mitwaba group to the southeast. To the northwest, the $\mathrm{S}_{1}$ fabric occurs within the Mitwaba group and is totally absent within the Mulumbi group lithologies (Fig. 2).

The Mulumbi group is made of: (1) the Kataba conglomerate containing pebbles from the underlying Mitwaba group metasedimentary rocks and related orthogneisses; (2) quartzites with conglomerate interca- lations; and (3) metapelites. The studied granitoids and the mafic igneous bodies do not intersect the Mulumbi group metasedimentary rocks. Therefore, the siliciclastic rocks of the Mulumbi group were deposited after $D_{1}$ and after the emplacement of the igneous rocks intruding the Mitwaba group. The Mulumbi group rocks are affected by the regional $\mathrm{D}_{2}$ Kibaran compressional structures, e.g. NE-trending isoclinal folds $\left(\mathrm{F}_{2}\right)$ and related axial planar foliation $\left(\mathrm{S}_{2}\right)$ forming the main physiography of the Kibaran orogenic system in the type-area. Mineral parageneses defining this NEtrending foliation indicate an increase in metamorphic grade from greenschist facies rocks in the northwest, to amphibolite facies to the southeast, including staurolite, kyanite and sillimanite-bearing metasedimentary rocks (e.g. Kokonyangi et al., 2001).

Granitic rocks exposed in the Mitwaba area (Fig. 2) form the Kifinga-Kisele batholith and subordinate satellite bodies, including Nyangwa, KungweKalumengongo, Kisandji and Fubwe plutons. Among them, the Kifinga-Kisele batholith, Nyangwa and Kungwe-Kalumengongo plutons were investigated for this study. The Kifinga-Kisele batholith includes 


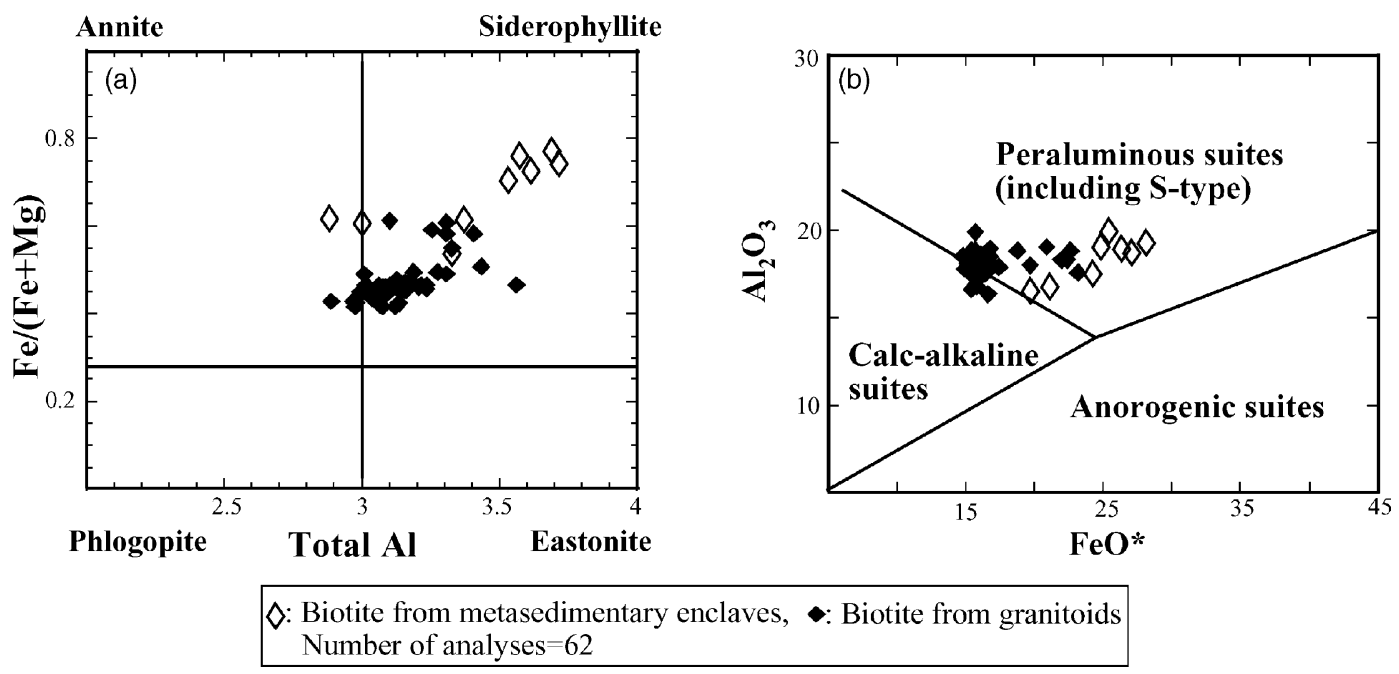

Fig. 3. (a) Composition of biotites from the Mitwaba granitoids in the diagram $\mathrm{Fe} /(\mathrm{Fe}+\mathrm{Mg}$ ) vs. Al. (b) Discrimination diagram for biotites from granitoids using $\mathrm{FeO}^{*}$ and $\mathrm{Al}_{2} \mathrm{O}_{3}$ (Abdel-Rahman, 1994). Most biotites from Mitwaba granitoids fall in the field for peraluminous S-type granitoids. See text for details.

three distinct plutons: (1) Kabonvia granodioritic augen gneiss; (2) Kisele monzogranite gneiss; and (3) Fwifwi foliated leucomonzogranite (Fig. 2). The main petrographic and mineralogical features of these intrusive bodies are summarized in Table 1. The biotites from Mitwaba granitoids display chemical characteristics of biotites from peraluminous granitoids (Fig. 3); their relatively high $\mathrm{Al}$ and low $\mathrm{Fe}$ contents are similar to those reported in peraluminous granites derived from partial melting of sedimentary rocks (Abdel-Rahman, 1994). Large euhedral muscovite crystals imbricated or in parallel growth with primary biotite and containing inclusion of accessory magmatic minerals contain high $\mathrm{Ti}, \mathrm{Fe}$ and $\mathrm{Si}$ atomic values pointing towards their magmatic origin (e.g. Speer, 1984). Ilmenite occurs in all granitoids and rutile $\left(99 \% \mathrm{TiO}_{2}\right)$ is included in biotite and its alteration product chlorite.

All the plutons were affected by $\mathrm{D}_{2}$ deformation event and related metamorphism $\mathrm{M}_{2}$. However, as stated above, the grade of $\mathrm{M}_{2}$ is variable within the belt; the Fwifwi Nyangwa and KungweKalumengongo plutons were metamorphosed under greenschist facies (chlorite zone) whereas the Kabonvia granodioritic augen gneiss (staurolite zone) and Kisele monzogranite gneiss (sillimanite zone) were metamorphosed under amphibolite facies P-T con- ditions. All the plutons exhibit sharp contact with Mitwaba group metasedimentary rocks and contain abundant foliated xenoliths of country rocks close to the contact.

Near the margins of the intrusive bodies, the $\mathrm{S}_{1}$ foliations in the granitoids and in the metasedimentary country rocks are parallel. These granitoids contain foliated biotite-muscovite rich restitic clots (surmicaceous enclaves of Didier, 1973). The central parts of the plutons show weaker foliation whereas the margins of the granitoid bodies display a stronger solid-state fabric (e.g. Fig. 2). The contact aureole includes porphyroblasts of biotite and garnet in metapelitic rocks and hornblende and garnet in mafic igneous rocks (Kokonyangi et al., 2001). Hornblende and garnet are strongly poikiloblastic and display pressure shadows developed during the main fabric-forming event $\mathrm{D}_{2}$. Some garnet porphyroblasts exhibit an internal fabric defined by quartz and opaque minerals, oblique to the $\mathrm{D}_{2}$ fabric. This internal fabric is related to an early event $\left(D_{1}\right)$, which was contemporaneous to the emplacement of the batholith.

The Kabonvia granodioritic augen gneiss forms an ENE-oriented orthogneiss body elongated parallel to the trend of the $\mathrm{D}_{1}$ fabric in the metasedimentary country rocks. A solid-state foliation $\left(S_{1}\right)$ is pronounced throughout this body and locally the 
Table 1

Summary of field and petrographic characteristics of Mitwaba granitoids

\begin{tabular}{|c|c|c|c|c|c|}
\hline & \multicolumn{3}{|c|}{ Kifinga-Kisele batholith (ca. $75 \mathrm{~km} \times 60 \mathrm{~km}$ ) } & \multirow[t]{2}{*}{ Nyangwa } & \multirow[t]{2}{*}{ Kungwe-Kalumengongo } \\
\hline & Kabonvia & Kisele & Fwifwi & & \\
\hline Deformation features & $\begin{array}{l}\mathrm{D}_{1} \text { and } \mathrm{F}_{1} \text { minor } \\
\text { folds, } \mathrm{D}_{2}\end{array}$ & $\begin{array}{l}D_{1}, D_{2} \text { and } F_{1} \\
\text { minor folds }\end{array}$ & $\begin{array}{l}D_{1}, D_{2} \text { and marginal } \\
\text { mylonitic foliation }\end{array}$ & $\begin{array}{l}\mathrm{D}_{1}, \mathrm{D}_{2}, \text { mesoscopic } \\
\text { folds }\end{array}$ & $\begin{array}{l}D_{1}, D_{2}, \text { minor shear } \\
\text { zones subparallel to } S_{2}\end{array}$ \\
\hline Contact aureole & Grt-Bt hornfels & N.O. & Grt-Hb hornfels & N.O. & N.O. \\
\hline $\begin{array}{l}\text { Regional metamorph. } \\
\text { zone of occurrence }\end{array}$ & Staurolite zone & Sillimanite zone & Grt-Bt-Chl zone & Chl zone & Grt-bt-Chl zone \\
\hline \multicolumn{6}{|l|}{ Mineralogy (vol.\%) } \\
\hline Alkali feldspar & $10-15\left(\mathrm{Or}_{92-96} \mathrm{Ab}_{7-3}\right)$ & $29-30\left(\mathrm{Or}_{95-96} \mathrm{Ab}_{5-4}\right)$ & $10-19\left(\mathrm{Or}_{93} \mathrm{Ab}_{6} \mathrm{An}_{1}\right)$ & $12-22\left(\mathrm{Or}_{98} \mathrm{Ab}_{2}\right)$ & $8-22\left(\mathrm{Or}_{74-89} \mathrm{Ab}_{26-11}\right)$ \\
\hline Plagioclase & $35-40\left(\mathrm{An}_{20-30} \mathrm{Ab}_{79-20}\right)$ & $\begin{array}{l}32-40\left(\mathrm{c}: \mathrm{An}_{12-13}\right. \\
\left.\mathrm{Ab}_{86} ; \mathrm{r}: \mathrm{An}_{2} \mathrm{Ab}_{98}\right)\end{array}$ & $\begin{array}{l}23-37\left(\mathrm{An}_{12-13}\right. \\
\left.\mathrm{Ab}_{81-85} \mathrm{Or}_{6-1}\right)\end{array}$ & $\begin{array}{l}25-35\left(\mathrm{An}_{7-10}\right. \\
\left.\mathrm{Ab}_{91-84} \mathrm{Or}_{1-6}\right)\end{array}$ & \\
\hline Biotite & $10-19\left(\mathrm{X}_{\mathrm{Mg}}: 0.50-0.53\right)$ & $3-6\left(\mathrm{X}_{\mathrm{Mg}}: 0.52\right)$ & 4-7 (X $\left.\mathrm{X}_{\mathrm{Mg}}: 0.41\right)$ & $\sim 10\left(\mathrm{X}_{\mathrm{Mg}}: 0.43-0.47\right)$ & $8-18\left(\mathrm{X}_{\mathrm{Mg}}: 0.42-0.43\right)$ \\
\hline Primary muscovite & $\sim 5\left(\mathrm{X}_{\mathrm{Mg}}: 0.62-0.66\right)$ & $1-4\left(\mathrm{X}_{\mathrm{Mg}}: 0.54\right)$ & $1-4\left(\mathrm{X}_{\mathrm{Mg}}: 0.52\right)$ & $1-2\left(X_{M g}: 0.60\right)$ & $2-3\left(\mathrm{X}_{\mathrm{Mg}}: 0.66\right)$ \\
\hline \multicolumn{6}{|l|}{ Accessories } \\
\hline Apatite & $\mathrm{xx}$ & $\mathrm{x}$ & $\mathrm{xx}$ & $\mathrm{xx}$ & $\mathrm{xx}$ \\
\hline Titanite & & & $\mathrm{x}$ & & $\mathrm{xx}$ \\
\hline Tourmaline & $\mathrm{x}$ & $\mathrm{xx}$ & $\mathrm{x}$ & $\mathrm{x}$ & $\mathrm{xx}$ \\
\hline Zircon & $\mathrm{xx}$ & $\mathrm{x}$ & $\mathrm{xx}$ & $\mathrm{xx}$ & $\mathrm{xx}$ \\
\hline Chlorite & $\mathrm{s}$ & $\mathrm{s}$ & $\mathrm{s}$ & $\mathrm{s}$ & $\mathrm{s}$ \\
\hline Musc/sericite & $\mathrm{s}$ & $\mathrm{s}$ & $\mathrm{s}$ & $\mathrm{s}$ & $\mathrm{s}$ \\
\hline Ilmenite & $\mathrm{xx}$ & $\mathrm{x}$ & $\mathrm{x}$ & $\mathrm{x}$ & $\mathrm{xx}$ \\
\hline Magnetite & $\mathrm{x}$ & & & & \\
\hline Rutile & $\mathrm{x}$ & $\mathrm{x}$ & & $\mathrm{x}$ & \\
\hline Metased. xenoliths & $\mathrm{xx}$ & $\mathrm{x}$ & $\mathrm{x}$ & $\mathrm{x}$ & $\mathrm{xx}$ \\
\hline Classification (QAP) & Granodiorite & Monzogranite & Monzogranite & Monzogranite & Monzogranite \\
\hline Inheritance & Zircon core & Zircon core & Zircon core & Zircon core & Zircon core \\
\hline Enclaves & $\begin{array}{l}\text { Surmicaceous } \\
\text { (Didier, 1973) }\end{array}$ & Surmicaceous & Surmicaceous & Surmicaceous & Surmicaceous \\
\hline
\end{tabular}

Notes. c: core; r: rim; x: present; xx: abundant; s: secondary; Grt: garnet; Bt: Biotite; Chl: Chlorite; Hb: Hornblende; N.O.: not observed; metased.: metasedimentary rocks; $\mathrm{XMg}=\mathrm{Mg} / \mathrm{Mg}+\mathrm{Fe}$; calculated in molar ratios. 
orthogneiss grades to banded migmatitic gneiss. The mineral paragenesis of sedimentary rocks metamorphosed along with this granitoid equilibrated in the staurolite zone (Kokonyangi et al., 2001). According to the classification of Cahen et al. (1984), this body belongs to the type A $\left(\mathrm{G}_{1}\right)$ granitoids supposedly emplaced at $1331 \pm 25 \mathrm{Ma}$.

The Kisele monzogranite gneiss is pink to pinkishgrey and contains enclaves of the Kabonvia granodiorite augen gneiss close to their mutual contact. This observation and geometrical relationships between these intrusions (Fig. 2) suggest that the Kisele monzogranite gneiss postdate the emplacement of the Kabonvia granodioritic augen gneiss. The Kisele monzogranite gneiss contains also surmicaceous enclaves and enclaves of metasedimentary rocks that could not be identified within the country rock successions and are inferred to represent material brought from the source of the granite. The mineral paragenesis (Table 1) of sedimentary rocks metamorphosed along with this granitoid equilibrated in the sillimanite zone. This granitoid displays crosscutting relations between $\mathrm{S}_{1}\left(70^{\circ} \mathrm{N}-80^{\circ} \mathrm{E}\right)$ and $\mathrm{S}_{2}\left(25^{\circ} \mathrm{N}-40^{\circ} \mathrm{E}\right)$ and therefore, according to Cahen et al. (1984), it can be classified as type $A$ or $\mathrm{G}_{1}$ Kibaran granite.

Compared to the Kisele monzogranite gneiss, the Fwifwi foliated leucomonzogranite is quartz-rich, weakly foliated in the centre and exhibits higher strain at the margin of the pluton where it grades to a gneissic facies. The western margin of the pluton is in contact with greenschist facies metamorphosed mafic igneous rocks intruding the Mitwaba group. In contrast to other plutons, this body contains titanite close to its contact with the mafic rocks (Fig. 2). However, away from the contact, titanite is absent. The Fwifwi foliated leucomonzogranite contains angular enclaves of the Kisele monzogranite gneiss along the contact between the two intrusive bodies. Pioneering conventional $\mathrm{Pb}-\mathrm{Pb}$ dating done almost half a century ago on this pluton (Eberhardt et al., 1956) yielded dates between $1050 \pm 50 \mathrm{Ma}$ and $1144 \pm 10 \mathrm{Ma}$. According to the above dates and based on the classification criteria of Cahen et al. (1984), the Fwifwi foliated leucomonzogranite belongs to type $\mathrm{C}\left(\mathrm{G}_{3}\right)$.

The Nyangwa pluton occurs to the northwest of the study area (Fig. 2). It is elongated $\mathrm{N} 30^{\circ} \mathrm{E}$, parallel to the trend of the regional $\mathrm{D}_{2}$ fabric. The pluton occurs within greenschist facies (chlorite-zone) metased- imentary rocks. The margins of the intrusion are buried beneath a Neoproterozoic and Phanerozoic sedimentary cover. The rocks are foliated, medium- to coarsegrained, and pinkish to greyish. Biotite flakes and Kfeldspar phenocrysts define the foliation. The pluton contains elongated elliptical enclaves of metasedimentary rocks distributed parallel to the foliation of the granite. Some quartz veins observed in the granite are isoclinally folded with the axial plane trending NE, parallel to the solid-state foliation in the granitoid and in the country rocks. No previous age determination was done on this pluton, but based on its regional NE elongation, it was inferred to be part of the ca. 1308 \pm 25 Ma type B $\left(G_{2}\right)$ granitoids (Cahen et al., 1984).

The Kungwe-Kalumengongo stock is a small granitic gneiss body intruding greenschist facies metasedimentary rocks. The granite is leucocratic, medium to fine grained, with sporadic K-feldspar phenocrysts. It is chilled against the Kalumengongo black shale formation (Mitwaba group) and contains abundant xenoliths of supracrustal metasedimentary country rocks. Metasedimentary enclaves observed in the central portion of this stock are unknown in the local lithostratigraphic sequence. The stock is particularly rich in tourmaline close to the contact with the country rocks. Two foliations are developed throughout this granitic body. The oldest trends $70^{\circ} \mathrm{N}-90^{\circ} \mathrm{E}$ and the youngest $20^{\circ} \mathrm{N}-30^{\circ} \mathrm{E}$ respectively, parallel to

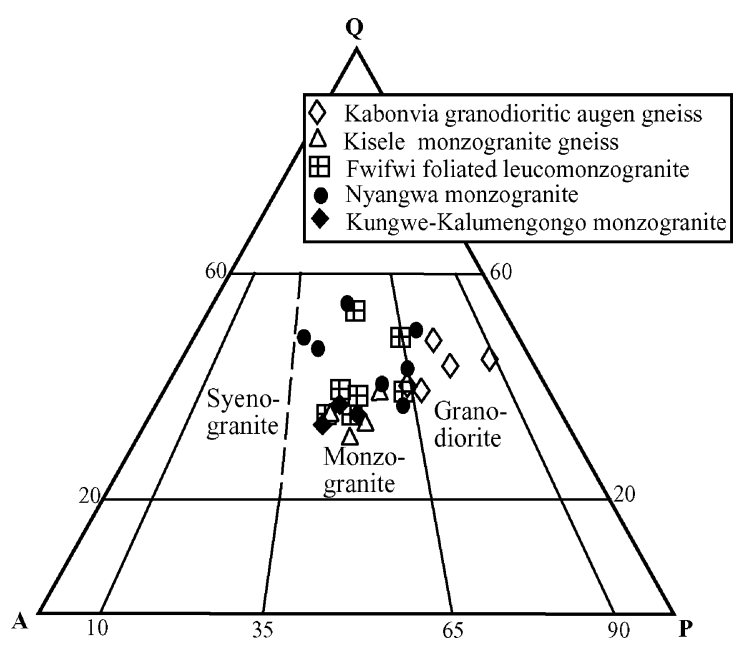

Fig. 4. Classification of the Mitwaba granitoids using the modal composition plotted in the quartz-alkali feldspar-plagioclase (Q-A-P) diagram of Streckeisen (1976). 
$S_{1}$ and $S_{2}$ in supracrustal country rocks. No previous age determination has been performed on this granitic stock, but according to Cahen et al. (1984), it would be classified as type $A$ or $G_{1}$ because it carries both foliations $\left(S_{1}\right.$ and $\left.S_{2}\right)$. The modal and mineralogical compositions of the Mitwaba granitoids are given in Table 1 and illustrated in Fig. 4. In this diagram, the Mitwaba granitoids fall in the fields of granodiorites for the Kabonvia pluton and monzogranites for the remaining plutons.

\section{Sampling and analytical procedures}

Ion Microprobe (SHRIMP) U-Pb zircon dating of representative samples from the Mitwaba granitoids (Table 2) was performed at the Research School of Earth Sciences (RSES), The Australian National University. Separated zircon grains were mounted in epoxy together with the RSES SHRIMP standard AS3 (Duluth Complex gabbroic anorthosite; Peaces and Miller, 1989) and SL13 (radiogenic ${ }^{206} \mathrm{~Pb} /{ }^{238} \mathrm{U}=$ 0.0928 and $238 \mathrm{ppm}$ U, Roddick and Van Breemen, 1994). Data have been reduced in a manner similar to that described by Compston et al. (1992) and Williams and Claesson (1987), and using the software SQUID (Ludwig, 2000). U/Pb in zircon unknowns were normalized to a ${ }^{206} \mathrm{~Pb}^{*} /{ }^{238} \mathrm{U}$ value of 0.1859 (equivalent to an age of $1099.1 \mathrm{Ma}$ ) for AS3. The $\mathrm{U}$ and Th concentrations were determined relative to those measured in the SL13 standard and the common Pb correction was determined from the measured ${ }^{204} \mathrm{~Pb}$ and using the appropriate common $\mathrm{Pb}$ isotopic composition assuming the Cumming and Richards (1975) model. The age calculations and statistical assessment of the data have been done utilizing the geochronological statistical software package Isoplot/Ex (version 2.00) of Ludwig (2000). Uncertainties in the isotopic ratios and ages in the data table (and in the error bars in the plotted data) are reported at $1 \sigma$, but where ages are calculated from combined data or from regression analysis the error quoted are $95 \%$ confidence limits. Where appropriate, Concordia Ages for some data sets are calculated using Ludwig (1998), again with 95\% confidence limit quoted for uncertainties.

Samples were powdered using an automatic agate mill at OCU. Two sets of new whole-rock chemical data are used in this study. The first set includes
21 whole-rock analyses determined at OCU using a Rigaku RIX-2000 X-ray fluorescence spectrometer on glass beads. Precision is $\sim 1 \%$ for major elements and $\sim 5 \%$ for trace elements. These data corresponds to analyses without rare-earth-element composition in Table 3. The second set consists of 17 remaining whole-rock analyses (Table 3) performed at Chemex Laboratories (Canada). Major element compositions were determined using an ICP-AES and trace elements were analysed using an ICP-MS. $\mathrm{Pb}, \mathrm{Ni}, \mathrm{Cr}$ and $\mathrm{Li}$ were analysed by AAS. The precisions are \pm 1 and $\pm 5 \%$ for major and trace elements, respectively. All analyses were recalculated to $100 \%$ anhydrous for inter-sample comparisons in the paper. The data were normalized to chondrite and primordial mantle using the reference values of Sun and McDonough (1989).

\section{Geochronology}

Most of the granite samples processed for this study yielded good quality zircons suitable for $\mathrm{U}-\mathrm{Pb}$ dating, with one sample (K2) producing the most complicated population. Inheritance, in the form of cores, is a common feature in the zircon grains from these granitoids. However, the use of cathodoluminescence imaging enables these xenocrystic components to be identified and generally avoided for this study mainly focussed on the crystallisation age of the granitoids.

\subsection{Kisele monzogranite gneiss}

The zircons from this sample are complicated by structure, alteration (through metamictisation), possible metamorphic rounding and the presence of metamorphic overgrowths. The general population comprises subhedral grains of various sizes that are yellow to brown, and translucent. Cathodoluminescence imaging shows strong concentric compositional zoning interpreted to represent the magmatic zircon, but with some grains showing large altered areas. A few grains have small, distinct overgrowths or zones that appear uniform (unzoned) in cathodoluminescence.

Twelve U-Th- $\mathrm{Pb}$ analyses were done on different zircons with the data reported in Table 2 and plotted on a conventional Wetherill U-Pb concordia shown in Fig. 5a. As anticipated, most analyses are highly 
Table 2

U-Pb SHRIMP isotopic data on zircons from Kibaran granitoids Mitwaba Katanga (Congo)

\begin{tabular}{|c|c|c|c|c|c|c|c|c|c|c|c|c|c|c|c|c|c|}
\hline \multirow{2}{*}{$\begin{array}{l}\text { Grain } \\
\text { spot }\end{array}$} & \multirow{2}{*}{$\begin{array}{l}\begin{array}{l}{ }^{206} \mathrm{~Pb}_{\mathrm{C}} \\
(\%)\end{array} \\
\text { granodion }\end{array}$} & \multirow{2}{*}{$\begin{array}{l}\mathrm{U} \\
\text { (ppm) } \\
\text { c augen }\end{array}$} & \multirow{2}{*}{$\begin{array}{l}\text { Th } \\
(\mathrm{ppm}) \\
\text { eiss }(\mathrm{K}\end{array}$} & \multirow{2}{*}{${ }^{232} \mathrm{Th} /{ }^{238} \mathrm{U}$} & \multirow[t]{2}{*}{$\begin{array}{l}{ }^{206} \mathrm{~Pb}^{*} \\
(\mathrm{ppm})\end{array}$} & \multirow[t]{2}{*}{$\begin{array}{l}\text { (1) }{ }^{207} \mathrm{~Pb}^{*} / \\
{ }^{206} \mathrm{~Pb}^{*}\end{array}$} & \multirow[t]{2}{*}{ $\pm \%$} & \multirow[t]{2}{*}{$\begin{array}{l}\text { (1) }{ }^{207} \mathrm{~Pb}^{*} / \\
{ }^{235} \mathrm{U}\end{array}$} & \multirow[t]{2}{*}{ $\pm \%$} & \multirow[t]{2}{*}{$\begin{array}{l}(1){ }^{206} \mathrm{~Pb}^{*} / \\
{ }^{238}{ }_{\mathrm{U}}\end{array}$} & \multirow[t]{2}{*}{ $\pm \%$} & \multirow[t]{2}{*}{ Err corr } & \multicolumn{2}{|c|}{$\begin{array}{l}\text { (1) }{ }^{206} \mathrm{~Pb} /{ }^{238} \mathrm{U} \\
\text { age (Ma) }\end{array}$} & \multicolumn{2}{|c|}{$\begin{array}{l}\text { (1) }{ }^{207} \mathrm{~Pb} /{ }^{206} \mathrm{~Pb} \\
\text { age (Ma) }\end{array}$} & \multirow[t]{2}{*}{$\begin{array}{l}\text { Dis- cordant } \\
(\%)\end{array}$} \\
\hline & & & & & & & & & & & & & & & & & \\
\hline 1.1 & 0.55 & 228 & 33 & 0.15 & 46.0 & 0.08654 & 0.93 & 2.785 & 1.2 & 0.2334 & 0.82 & 0.660 & 1352.20 & \pm 10.0 & 1350 & \pm 18 & 0 \\
\hline 2.1 & 0.94 & 256 & 56 & 0.23 & 52.1 & 0.0890 & 3.5 & 2.88 & 3.6 & 0.2347 & 0.75 & 0.208 & 1359.10 & \pm 9.2 & 1404 & \pm 68 & 3 \\
\hline 3.1 & 0.07 & 635 & 46 & 0.07 & 135 & 0.08829 & 0.33 & 2.998 & 0.73 & 0.2463 & 0.65 & 0.895 & 1419.10 & \pm 8.3 & 1388.8 & \pm 6.3 & -2 \\
\hline 4.1 & 3.91 & 376 & 42 & 0.12 & 73.6 & 0.0892 & 1.7 & 2.695 & 1.9 & 0.2191 & 0.70 & 0.377 & 1277.00 & \pm 8.1 & 1409 & \pm 33 & 9 \\
\hline 5.1 & 0.28 & 214 & 42 & 0.20 & 43.9 & 0.08665 & 0.72 & 2.851 & 1.0 & 0.2386 & 0.75 & 0.725 & 1379.30 & \pm 9.3 & 1353 & \pm 14 & -2 \\
\hline 6.1 & 0.46 & 251 & 63 & 0.26 & 53.3 & 0.08909 & 1.0 & 3.021 & 1.3 & 0.2459 & 0.83 & 0.632 & 1418.00 & \pm 11 & 1406 & \pm 19 & -1 \\
\hline 7.1 & 0.44 & 179 & 20 & 0.12 & 37.6 & 0.08729 & 0.97 & 2.922 & 1.2 & 0.2428 & 0.78 & 0.625 & 1401.00 & \pm 9.8 & 1367 & \pm 19 & -2 \\
\hline 8.1 & 1.00 & 88 & 148 & 1.74 & 26.3 & 0.1182 & 1.2 & 5.634 & 1.6 & 0.3457 & 1.0 & 0.659 & 1914.00 & \pm 17 & 1929 & \pm 21 & 1 \\
\hline 8.2 & 0.13 & 286 & 28 & 0.10 & 60.7 & 0.08873 & 0.69 & 3.019 & 0.98 & 0.2467 & 0.70 & 0.714 & 1421.60 & \pm 8.9 & 1398 & \pm 13 & -2 \\
\hline 9.1 & 0.20 & 420 & 41 & 0.10 & 85.6 & 0.08873 & 0.58 & 2.898 & 0.89 & 0.2369 & 0.68 & 0.756 & 1370.40 & \pm 8.3 & 1398 & \pm 11 & 2 \\
\hline 10.1 & 0.33 & 236 & 36 & 0.16 & 47.7 & 0.08795 & 0.79 & 2.839 & 1.1 & 0.2341 & 0.72 & 0.677 & 1356.10 & \pm 8.8 & 1381 & \pm 15 & 2 \\
\hline 11.1 & 0.22 & 369 & 48 & 0.13 & 76.0 & 0.08774 & 0.57 & 2.892 & 0.89 & 0.2391 & 0.68 & 0.770 & 1381.80 & \pm 8.5 & 1377 & \pm 11 & 0 \\
\hline 12.1 & 0.43 & 283 & 46 & 0.17 & 57.9 & 0.08720 & 0.95 & 2.850 & 1.2 & 0.2370 & 0.71 & 0.600 & 1371.30 & \pm 8.8 & 1365 & \pm 18 & 0 \\
\hline 13.1 & 2.54 & 161 & 45 & 0.29 & 30.7 & 0.0927 & 2.2 & 2.765 & 2.3 & 0.2162 & 0.77 & 0.330 & 1261.80 & \pm 8.9 & 1483 & \pm 42 & 15 \\
\hline 14.1 & 0.42 & 146 & 64 & 0.45 & 30.2 & 0.08885 & 1.0 & 2.943 & 1.4 & 0.2402 & 1.0 & 0.718 & 1388.00 & \pm 13 & 1401 & \pm 19 & 1 \\
\hline 15.1 & 0.59 & 213 & 30 & 0.14 & 43.9 & 0.08717 & 0.94 & 2.872 & 1.3 & 0.2390 & 0.87 & 0.679 & 1381.00 & \pm 11 & 1364 & \pm 18 & -1 \\
\hline Kisele $n$ & tonzogranit & gneiss $(1$ & & & & & & & & & & & & & & & \\
\hline 1.1 & 0.9 & 536 & 107 & 0.21 & 75.8 & 0.08529 & 0.76 & 1.92 & 1 & 0.1632 & 0.7 & 0.679 & 974.70 & \pm 6.4 & 1322.00 & \pm 15 & 26 \\
\hline 2.1 & 0.32 & 2436 & 318 & 0.13 & 227 & 0.07736 & 0.44 & 1.151 & 0.94 & 0.10794 & 0.83 & 0.882 & 660.80 & \pm 5.2 & 1130.50 & \pm 8.9 & 42 \\
\hline 3.1 & 0.36 & 1562 & 23 & 0.02 & 224 & 0.07536 & 0.37 & 1.727 & 0.73 & 0.1662 & 0.63 & 0.864 & 991.00 & \pm 5.8 & 1078.00 & \pm 7.4 & 8 \\
\hline 4.1 & 0.14 & 430 & 72 & 0.17 & 89.2 & 0.08774 & 0.61 & 2.921 & 1 & 0.2415 & 0.81 & 0.798 & 1394.00 & \pm 10 & 1377.00 & \pm 12 & -1 \\
\hline 5.1 & 0.14 & 350 & 146 & 0.43 & 72.6 & 0.08792 & 0.61 & 2.92 & 0.91 & 0.2409 & 0.68 & 0.749 & 1391.30 & \pm 8.6 & 1381.00 & \pm 12 & -1 \\
\hline 6.1 & 5.29 & 2504 & 144 & 0.06 & 184 & 0.075 & 4 & 0.837 & 4.1 & 0.08087 & 0.72 & 0.177 & 501.30 & \pm 3.5 & 1070.00 & \pm 81 & 53 \\
\hline 7.1 & 0.13 & 388 & 43 & 0.11 & 79.7 & 0.08834 & 0.46 & 2.911 & 0.82 & 0.239 & 0.68 & 0.826 & 1381.50 & \pm 8.4 & 1390.00 & \pm 8.9 & 1 \\
\hline 8.1 & 1.13 & 6647 & 1269 & 0.2 & 162 & 0.05916 & 0.99 & 0.2294 & 1.2 & 0.02813 & 0.64 & 0.542 & 178.80 & \pm 1.1 & 573.00 & \pm 22 & 69 \\
\hline 9.1 & 0.23 & 861 & 23 & 0.03 & 134 & 0.07542 & 0.43 & 1.875 & 0.79 & 0.1804 & 0.66 & 0.841 & 1068.90 & \pm 6.5 & 1079.70 & \pm 8.6 & 1 \\
\hline 10.1 & 2.51 & 11334 & 3042 & 0.28 & 233 & 0.05958 & 1.3 & 0.1917 & 1.4 & 0.02334 & 0.68 & 0.473 & 148.70 & \pm 1.0 & 588.00 & \pm 27 & 75 \\
\hline 11.1 & 0.7 & 5555 & 1447 & 0.27 & 123 & 0.06658 & 0.86 & 0.2349 & 1.1 & 0.02559 & 0.63 & 0.593 & 162.90 & \pm 1.0 & 825.00 & \pm 18 & 80 \\
\hline 12.1 & 1.39 & 9865 & 3610 & 0.38 & 210 & 0.05912 & 1.1 & 0.1991 & 1.5 & 0.02443 & 1 & 0.683 & 155.60 & \pm 1.6 & 572.00 & \pm 24 & 73 \\
\hline Fwifwi & oliated leu & monzogra & ite (K2: & & & & & & & & & & & & & & \\
\hline 1.1 & 0.64 & 158 & 52 & 0.34 & 32.6 & 0.08687 & 1.1 & 2.852 & 1.3 & 0.2381 & 0.77 & 0.576 & 1377.10 & \pm 9.6 & 1358.00 & \pm 21 & -1 \\
\hline 2.1 & 0.66 & 127 & 33 & 0.27 & 26.7 & 0.0874 & 1.2 & 2.931 & 1.4 & 0.2433 & 0.8 & 0.559 & 1404.00 & \pm 10 & 1369.00 & \pm 23 & -3 \\
\hline 3.1 & 0.41 & 159 & 51 & 0.33 & 32.9 & 0.08887 & 0.97 & 2.937 & 1.2 & 0.2397 & 0.76 & 0.619 & 1385.10 & \pm 9.5 & 1401.00 & \pm 19 & 1 \\
\hline 4.1 & 0.14 & 144 & 50 & 0.36 & 30.1 & 0.09089 & 0.88 & 3.041 & 1.2 & 0.2426 & 0.78 & 0.663 & 1400.30 & \pm 9.8 & 1444.00 & \pm 17 & 3 \\
\hline 5.1 & 0.49 & 149 & 47 & 0.33 & 31 & 0.08683 & 1.1 & 2.876 & 1.5 & 0.2402 & 1.1 & 0.699 & 1388.00 & \pm 13 & 1357.00 & \pm 21 & -2 \\
\hline 6.1 & 0.29 & 205 & 60 & 0.3 & 42.3 & 0.08862 & 0.77 & 2.92 & 1.1 & 0.239 & 0.75 & 0.7 & 1381.60 & \pm 9.4 & 1396.00 & \pm 15 & 1 \\
\hline 7.1 & 0.36 & 226 & 60 & 0.28 & 47.1 & 0.0877 & 0.8 & 2.922 & 1.1 & 0.2416 & 0.72 & 0.669 & 1395.20 & \pm 9.1 & 1376.00 & \pm 15 & -1 \\
\hline 8.1 & 0.43 & 155 & 49 & 0.32 & 31.8 & 0.08767 & 1.1 & 2.868 & 1.5 & 0.2373 & 1 & 0.68 & 1372.00 & \pm 12 & 1375.00 & \pm 21 & 0 \\
\hline 9.1 & 0.47 & 155 & 53 & 0.35 & 31.9 & 0.08676 & 1.1 & 2.846 & 1.3 & 0.2379 & 0.77 & 0.588 & 1375.60 & \pm 9.5 & 1355.00 & \pm 20 & -2 \\
\hline 10.1 & 0.45 & 171 & 56 & 0.34 & 35.4 & 0.0875 & 0.97 & 2.898 & 1.3 & 0.2402 & 0.79 & 0.63 & 1387.60 & \pm 9.8 & 1372.00 & \pm 19 & -1 \\
\hline 11.1 & 0.55 & 165 & 57 & 0.36 & 34 & 0.08648 & 1.1 & 2.841 & 1.5 & 0.2383 & 0.97 & 0.657 & 1378.00 & \pm 12 & 1349.00 & \pm 21 & -2 \\
\hline 12.1 & 0.28 & 234 & 56 & 0.25 & 48.6 & 0.08747 & 0.7 & 2.909 & 1 & 0.2412 & 0.72 & 0.717 & 1393.10 & \pm 9.0 & 1371.00 & \pm 13 & -2 \\
\hline 13.1 & 0.47 & 181 & 58 & 0.33 & 38.1 & 0.08735 & 0.97 & 2.94 & 1.2 & 0.2442 & 0.75 & 0.613 & 1408.20 & \pm 9.5 & 1368.00 & \pm 19 & -3 \\
\hline 14.1 & 0.65 & 140 & 49 & 0.36 & 29.2 & 0.087 & 1.2 & 2.903 & 1.6 & 0.242 & 1.1 & 0.661 & 1397.00 & \pm 13 & 1360.00 & \pm 23 & -3 \\
\hline 15.1 & 0.78 & 114 & 30 & 0.27 & 23.4 & 0.0876 & 1.4 & 2.857 & 1.6 & 0.2365 & 0.85 & 0.52 & 1369.00 & \pm 11 & 1374.00 & \pm 27 & 0 \\
\hline 16.1 & 0.67 & 143 & 44 & 0.32 & 30.4 & 0.08715 & 1.1 & 2.953 & 1.5 & 0.2458 & 0.93 & 0.631 & 1417.00 & \pm 12 & 1364.00 & \pm 22 & -4 \\
\hline
\end{tabular}


Table 2 (Continued)

\begin{tabular}{|c|c|c|c|c|c|c|c|c|c|c|c|c|c|c|c|c|c|}
\hline \multirow{2}{*}{$\begin{array}{l}\text { Grain } \\
\text { spot } \\
\text { Kungwe- }\end{array}$} & \multirow{2}{*}{$\begin{array}{l}\begin{array}{l}{ }^{206} \mathrm{~Pb}_{\mathrm{C}} \\
(\%)\end{array} \\
\text { Kalumengo }\end{array}$} & \multirow{2}{*}{$\begin{array}{l}\mathrm{U} \\
(\mathrm{ppm}) \\
\text { ro monzo }\end{array}$} & \multirow{2}{*}{$\begin{array}{l}\text { Th } \\
(\mathrm{ppm}) \\
\text { anite }(\mathrm{K}\end{array}$} & \multirow{2}{*}{${ }^{232} \mathrm{Th} /{ }^{238} \mathrm{U}$} & \multirow[t]{2}{*}{$\begin{array}{l}{ }^{206} \mathrm{~Pb}^{*} \\
(\mathrm{ppm})\end{array}$} & \multirow[t]{2}{*}{$\begin{array}{l}\text { (1) }{ }^{207} \mathrm{~Pb}^{*} / \\
206 \mathrm{~Pb}^{*}\end{array}$} & \multirow[t]{2}{*}{ $\pm \%$} & \multirow[t]{2}{*}{$\begin{array}{l}{ }^{(1)}{ }^{207} \mathrm{~Pb}^{*} / \\
{ }^{235} \mathrm{U}\end{array}$} & \multirow[t]{2}{*}{ $\pm \%$} & \multirow[t]{2}{*}{$\begin{array}{l}{ }^{(1)}{ }^{206} \mathrm{~Pb}^{*} / \\
{ }^{238} \mathrm{U}\end{array}$} & \multirow[t]{2}{*}{ $\pm \%$} & \multirow[t]{2}{*}{ Err corr } & \multicolumn{2}{|c|}{$\begin{array}{l}\text { (1) }{ }^{206} \mathrm{~Pb} /{ }^{238} \mathrm{U} \\
\text { age (Ma) }\end{array}$} & \multicolumn{2}{|c|}{$\begin{array}{l}\text { (1) }{ }^{207} \mathrm{~Pb} /{ }^{206} \mathrm{~Pb} \\
\text { age (Ma) }\end{array}$} & \multirow[t]{2}{*}{$\begin{array}{l}\text { Dis- cordant } \\
(\%)\end{array}$} \\
\hline & & & & & & & & & & & & & & & & & \\
\hline 1.1 & 0.35 & 133 & 41 & 0.32 & 27.2 & 0.08778 & 1 & 2.877 & 1.3 & 0.2377 & 0.82 & 0.616 & 1375.00 & \pm 10 & 1378.00 & \pm 20 & 0 \\
\hline 2.1 & 0.78 & 219 & 76 & 0.36 & 39.9 & 0.08807 & 1.1 & 2.557 & 1.3 & 0.2106 & 0.73 & 0.552 & 1232.00 & \pm 8.2 & 1384.00 & \pm 21 & 11 \\
\hline 4.1 & 0.21 & 315 & 81 & 0.26 & 64.9 & 0.08766 & 0.64 & 2.891 & 0.95 & 0.2392 & 0.7 & 0.736 & 1382.30 & \pm 8.7 & 1375.00 & \pm 12 & -1 \\
\hline 5.1 & 0.42 & 199 & 62 & 0.32 & 41.6 & 0.08681 & 0.92 & 2.902 & 1.2 & 0.2424 & 0.74 & 0.627 & 1399.30 & \pm 9.3 & 1356.00 & \pm 18 & -3 \\
\hline 6.1 & 0.35 & 129 & 65 & 0.52 & 26.9 & 0.0892 & 1.2 & 2.966 & 1.4 & 0.2412 & 0.81 & 0.562 & 1393.00 & \pm 10 & 1408.00 & \pm 23 & 1 \\
\hline 7.1 & 0.3 & 172 & 62 & 0.37 & 35.6 & 0.08871 & 0.89 & 2.935 & 1.2 & 0.24 & 0.76 & 0.649 & 1386.70 & \pm 9.4 & 1398.00 & \pm 17 & 1 \\
\hline 8.1 & 0.5 & 174 & 57 & 0.34 & 36 & 0.08604 & 1.1 & 2.846 & 1.3 & 0.2399 & 0.75 & 0.578 & 1386.20 & \pm 9.4 & 1339.00 & \pm 21 & -4 \\
\hline 10.1 & 0.56 & 139 & 48 & 0.35 & 29.6 & 0.0879 & 1.1 & 2.977 & 1.5 & 0.2457 & 0.89 & 0.613 & 1416.00 & \pm 11 & 1379.00 & \pm 22 & -3 \\
\hline 11.1 & 0.37 & 178 & 58 & 0.34 & 37.6 & 0.08634 & 1.1 & 2.922 & 1.3 & 0.2455 & 0.75 & 0.578 & 1415.10 & \pm 9.6 & 1346.00 & \pm 21 & -5 \\
\hline 12.1 & 0.54 & 118 & 44 & 0.39 & 24.9 & 0.0874 & 1.6 & 2.94 & 1.8 & 0.244 & 0.82 & 0.465 & 1407.00 & \pm 10 & 1369.00 & \pm 30 & -3 \\
\hline 13.1 & 0.14 & 173 & 57 & 0.34 & 36.2 & 0.08845 & 0.96 & 2.97 & 1.2 & 0.2435 & 0.76 & 0.618 & 1404.90 & \pm 9.6 & 1392.00 & \pm 18 & -1 \\
\hline 14.1 & 0.31 & 159 & 51 & 0.33 & 33.3 & 0.089 & 1.1 & 2.984 & 1.4 & 0.2431 & 0.77 & 0.558 & 1403.00 & \pm 9.7 & 1405.00 & \pm 22 & 0 \\
\hline 15.1 & 0.33 & 198 & 54 & 0.28 & 41.6 & 0.08805 & 0.85 & 2.963 & 1.1 & 0.244 & 0.74 & 0.658 & 1407.60 & \pm 9.4 & 1384.00 & \pm 16 & -2 \\
\hline \multicolumn{18}{|c|}{ Nyangwa monzogranite (K30) } \\
\hline 1.1 & 0.13 & 429 & 108 & 0.26 & 80.6 & 0.08885 & 0.62 & 2.673 & 0.92 & 0.2182 & 0.69 & 0.745 & 1272.20 & \pm 7.9 & 1401.00 & \pm 12 & 9 \\
\hline 2.1 & 0.32 & 343 & 41 & 0.12 & 70.4 & 0.08778 & 0.72 & 2.884 & 1.6 & 0.2383 & 1.4 & 0.891 & 1378.00 & \pm 18 & 1378.00 & \pm 14 & 0 \\
\hline 3.1 & 0.32 & 215 & 67 & 0.32 & 45 & 0.08788 & 0.79 & 2.946 & 1.2 & 0.2432 & 0.95 & 0.77 & 1403.00 & \pm 12 & 1380.00 & \pm 15 & -2 \\
\hline 4.1 & 0.13 & 263 & 104 & 0.41 & 53.8 & 0.08861 & 0.6 & 2.904 & 1.1 & 0.2377 & 0.91 & 0.836 & 1375.00 & \pm 11 & 1396.00 & \pm 11 & 2 \\
\hline 6.1 & 0.22 & 450 & 52 & 0.12 & 91.6 & 0.08786 & 0.51 & 2.864 & 1.1 & 0.2364 & 0.92 & 0.875 & 1368.00 & \pm 11 & 1379.50 & \pm 9.8 & 1 \\
\hline 7.1 & 0.33 & 272 & 95 & 0.36 & 56.7 & 0.08859 & 0.73 & 2.957 & 1.1 & 0.2421 & 0.88 & 0.768 & 1398.00 & \pm 11 & 1395.00 & \pm 14 & 0 \\
\hline 8.1 & 0.18 & 389 & 43 & 0.11 & 80.3 & 0.0871 & 0.49 & 2.882 & 0.91 & 0.24 & 0.77 & 0.842 & 1386.70 & \pm 9.6 & 1362.60 & \pm 9.5 & -2 \\
\hline 9.1 & 0.31 & 476 & 66 & 0.14 & 98 & 0.08814 & 0.5 & 2.905 & 0.83 & 0.239 & 0.67 & 0.798 & 1381.50 & \pm 8.3 & 1385.60 & \pm 9.6 & 0 \\
\hline 10.1 & 2.43 & 305 & 36 & 0.12 & 60.7 & 0.0884 & 3.3 & 2.753 & 3.4 & 0.2259 & 0.88 & 0.26 & 1313.00 & \pm 11 & 1391.00 & \pm 63 & 6 \\
\hline 11.1 & 0.23 & 128 & 44 & 0.36 & 26.2 & 0.08953 & 0.97 & 2.933 & 1.3 & 0.2376 & 0.83 & 0.648 & 1374.00 & \pm 10 & 1416.00 & \pm 19 & 3 \\
\hline 12.1 & 0.36 & 329 & 122 & 0.38 & 64 & 0.08696 & 0.64 & 2.705 & 0.94 & 0.2256 & 0.69 & 0.731 & 1311.40 & \pm 8.2 & 1360.00 & \pm 12 & 4 \\
\hline 13.1 & 0.14 & 329 & 40 & 0.13 & 68.9 & 0.08747 & 0.56 & 2.938 & 1.1 & 0.2436 & 0.99 & 0.871 & 1406.00 & \pm 13 & 1371.00 & \pm 11 & -3 \\
\hline 14.1 & 0.18 & 295 & 56 & 0.2 & 60.8 & 0.08756 & 0.57 & 2.886 & 1 & 0.239 & 0.84 & 0.829 & 1382.00 & \pm 10 & 1373.00 & \pm 11 & -1 \\
\hline 15.1 & 0.5 & 316 & 99 & 0.32 & 61.1 & 0.08659 & 0.74 & 2.676 & 1.1 & 0.2242 & 0.8 & 0.736 & 1303.80 & \pm 9.4 & 1351.00 & \pm 14 & 4 \\
\hline 16.1 & 0.05 & 325 & 30 & 0.1 & 66.8 & 0.08828 & 0.5 & 2.912 & 0.87 & 0.2392 & 0.71 & 0.817 & 1382.80 & \pm 8.8 & 1388.60 & \pm 9.6 & 0 \\
\hline
\end{tabular}

Errors are $1 \sigma ; \mathrm{Pbc}$ and $\mathrm{Pb}^{*}$ indicate the common and radiogenic portions, respectively. Error in standard calibration was $0.19 \%$ (not included in above errors but required when comparing data from different mounts). (1) Common $\mathrm{Pb}$ corrected using measured ${ }^{204} \mathrm{~Pb}$. 
Table 3

Representative major and trace element compositions of Mitwaba granitoids (Katanga, Congo)

\begin{tabular}{|c|c|c|c|c|c|c|c|c|c|c|c|c|c|c|}
\hline & \multicolumn{14}{|l|}{ Plutons } \\
\hline & \multicolumn{6}{|l|}{ Kabonvia } & \multicolumn{6}{|l|}{ Kisele } & \multicolumn{2}{|l|}{ Fwifwi } \\
\hline & K12.1 & $\mathrm{K} 12.2$ & K12.3 & K12.4 & $\mathrm{K} 12.5$ & K12.6 & K2.1 & $\mathrm{K} 2.2$ & $\mathrm{~K} 2.3$ & K2.4 & $\mathrm{K} 2.5$ & K2.6 & K23.1 & K23.2 \\
\hline $\mathrm{SiO}_{2}$ & 69.39 & 68.32 & 67.69 & 69.78 & 67.35 & 68.68 & 74.16 & 75.46 & 74.68 & 75.45 & 73.35 & 74.88 & 70.84 & 74.21 \\
\hline $\mathrm{TiO}_{2}$ & 0.51 & 0.53 & 0.46 & 0.51 & 0.52 & 0.47 & 0.17 & 0.16 & 0.17 & 0.18 & 0.19 & 0.18 & 0.48 & 0.17 \\
\hline $\mathrm{Al}_{2} \mathrm{O}_{3}$ & 13.79 & 14.25 & 13.06 & 14.57 & 14.49 & 13.68 & 13.7 & 12.66 & 12.84 & 14.36 & 14.97 & 13.27 & 13.71 & 12.35 \\
\hline $\mathrm{Fe}_{2} \mathrm{O}_{3}$ & 3.47 & 3.82 & 3.71 & 3.46 & 3.74 & 3.77 & 1.44 & 1.22 & 1.23 & 1.48 & 1.19 & 1.22 & 2.95 & 1.46 \\
\hline $\mathrm{MnO}$ & 0.07 & 0.07 & 0.04 & 0.07 & 0.07 & 0.04 & 0.03 & 0.02 & 0.02 & 0.03 & 0.02 & 0.02 & 0.03 & 0.01 \\
\hline $\mathrm{MgO}$ & 1.66 & 1.72 & 1.67 & 1.74 & 1.82 & 1.85 & 0.57 & 0.49 & 0.51 & 0.63 & 0.51 & 0.51 & 1.01 & 0.41 \\
\hline $\mathrm{CaO}$ & 1.36 & 1.32 & 1.95 & 1.34 & 1.31 & 1.95 & 0.52 & 0.58 & 0.68 & 0.57 & 0.58 & 0.58 & 2.12 & 0.72 \\
\hline $\mathrm{Na}_{2} \mathrm{O}$ & 2.68 & 2.66 & 1.72 & 2.72 & 2.66 & 1.76 & 4.55 & 3.49 & 3.87 & 4.80 & 3.48 & 3.58 & 2.25 & 1.78 \\
\hline $\mathrm{K}_{2} \mathrm{O}$ & 4.71 & 5.03 & 4.4 & 4.64 & 4.85 & 4.34 & 2.93 & 4.43 & 3.82 & 2.95 & 4.22 & 4.35 & 5.05 & 6.46 \\
\hline $\mathrm{P}_{2} \mathrm{O}_{5}$ & 0.12 & 0.13 & 0.1 & 0.12 & 0.14 & 0.11 & 0.08 & 0.09 & 0.08 & 0.07 & 0.08 & 0.08 & 0.18 & 0.06 \\
\hline LOI & 1.87 & 1.74 & 3.34 & 1.49 & 1.094 & 1.55 & 1.4 & 1.04 & 1.5 & 1.02 & 0.98 & 1.01 & 1.37 & 2.27 \\
\hline Total & 99.76 & 99.71 & 98.26 & 100.44 & 98.04 & 98.20 & 99.59 & 99.68 & 99.43 & 101.54 & 99.56 & 99.69 & 100.1 & 99.95 \\
\hline Cs & 18.2 & 19.6 & 6.8 & & & & 1.3 & 1.3 & 1.2 & & & & 4.2 & 11.8 \\
\hline $\mathrm{Rb}$ & 309 & 329 & 225 & 255 & 268 & 186 & 132 & 185.5 & 168 & 119 & 151 & 158 & 254 & 249 \\
\hline $\mathrm{Ba}$ & 999 & 1085 & 836 & 909 & 958 & 771 & 242 & 384 & 332 & 229 & 338 & 342 & 947 & 375 \\
\hline Th & 14 & 16 & 17 & & & & 16 & 20 & 25 & & & & 17 & 27 \\
\hline $\mathrm{U}$ & 3 & 3.5 & 4 & & & & 13 & 16 & 19 & & & & 3 & 7.5 \\
\hline $\mathrm{Ta}$ & 3 & 4 & 3 & & & & 6 & 6 & 6 & & & & 6 & 9 \\
\hline $\mathrm{Nb}$ & 13 & 14 & 13 & 12 & 12 & 11 & 9 & 9 & 10 & 7 & 6 & 6 & 15 & 16 \\
\hline $\mathrm{Sr}$ & 160.5 & 159 & 226 & 141 & 140 & 196 & 65.8 & 72.6 & 74.5 & 61 & 61 & 63 & 284 & 183 \\
\hline $\mathrm{Hf}$ & 5 & 6 & 6 & & & & 5 & 4 & 6 & & & & 6 & 3 \\
\hline $\mathrm{Zr}$ & 279 & 281 & 296 & 177 & 185 & 207 & 267 & 241 & 278 & 172 & 126 & 131 & 300 & 192 \\
\hline $\mathrm{Y}$ & 26 & 34 & 28.5 & 27 & 33 & 30 & 20 & 43.5 & 52 & 22 & 43 & 42 & 35.5 & 46.5 \\
\hline V & 65 & 70 & 60 & 60.2 & 63.2 & 59.8 & 10 & 20 & 25 & 19.9 & 17.8 & 20.1 & 50 & 15 \\
\hline $\mathrm{Cr}$ & 64 & 64 & 56 & 70 & 70 & 56 & 4 & 6 & 4 & 8 & 7 & 8 & 46 & 2 \\
\hline $\mathrm{Ni}$ & 30 & 30 & 25 & 21 & 22 & 18 & 15 & 15 & 10 & 5 & 7 & 7 & 10 & 10 \\
\hline $\mathrm{Cu}$ & 25 & 25 & 25 & 20 & 16 & 17 & 15 & 10 & 5 & 1 & 2 & 3 & 5 & 10 \\
\hline $\mathrm{Pb}$ & 20 & 20 & 7 & 31 & 32 & 12 & 7 & 9 & 11 & 18 & 24 & 24 & 7 & 9 \\
\hline $\mathrm{Zn}$ & 75 & 85 & 50 & 62 & 66 & 28 & 30 & 20 & 30 & 12 & 10 & 10 & 25 & 40 \\
\hline Co & 104 & 71 & 109.5 & & & & 96 & 103 & 67 & & & & 66.5 & 150.5 \\
\hline $\mathrm{La}$ & 50 & 52 & 56.5 & & & & 41.5 & 44 & 57.5 & & & & 50.5 & 59.5 \\
\hline $\mathrm{Ce}$ & 96 & 105 & 112 & & & & 83 & 87.5 & 112.5 & & & & 104.5 & 129 \\
\hline $\operatorname{Pr}$ & 11 & 12.5 & 13 & & & & 9.4 & 9.8 & 12.5 & & & & 12.6 & 15.7 \\
\hline $\mathrm{Nd}$ & 41 & 46 & 44.5 & & & & 31.5 & 34.5 & 44.5 & & & & 46.5 & 58.5 \\
\hline $\mathrm{Sm}$ & 7.5 & 8.2 & 8 & & & & 6.2 & 7.3 & 8.8 & & & & 8.9 & 12 \\
\hline $\mathrm{Eu}$ & 1.4 & 1.4 & 1.3 & & & & 0.9 & 1 & 1.1 & & & & 1.6 & 1 \\
\hline $\mathrm{Gd}$ & 6.7 & 7.1 & 6 & & & & 4.9 & 6.9 & 8.4 & & & & 7.2 & 9.9 \\
\hline $\mathrm{Tb}$ & 1 & 1.1 & 0.9 & & & & 0.8 & 1.2 & 1.5 & & & & 1.1 & 1.4 \\
\hline
\end{tabular}




\begin{tabular}{|c|c|c|c|c|c|c|c|c|c|c|c|c|c|c|}
\hline & \multicolumn{14}{|l|}{ Plutons } \\
\hline & \multicolumn{6}{|c|}{ Kabonvia } & \multicolumn{6}{|l|}{ Kisele } & \multicolumn{2}{|l|}{ Fwifwi } \\
\hline & K12.1 & $\mathrm{K} 12.2$ & K12.3 & K12.4 & K12.5 & K12.6 & K2.1 & K 2.2 & K 2.3 & K2.4 & K2.5 & K2.6 & K23.1 & K23.2 \\
\hline Dy & 5.2 & 6.5 & 4.8 & & & & 4.7 & 7.9 & 9.5 & & & & 6 & 8.9 \\
\hline Ho & 0.9 & 1.2 & 1.1 & & & & 0.8 & 1.6 & 1.9 & & & & 1.2 & 1.6 \\
\hline $\mathrm{Er}$ & 3 & 3.7 & 3 & & & & 2.2 & 4.9 & 5.7 & & & & 3.6 & 4.7 \\
\hline $\mathrm{Tm}$ & 0.4 & 0.6 & 0.4 & & & & 0.3 & 0.7 & 0.9 & & & & 0.5 & 0.6 \\
\hline $\mathrm{Yb}$ & 2.6 & 3.8 & 3.3 & & & & 2 & 5.2 & 6.2 & & & & 3.2 & 4.1 \\
\hline $\mathrm{Lu}$ & 0.4 & 0.5 & 0.4 & & & & 0.3 & 0.8 & 1 & & & & 0.5 & 0.6 \\
\hline $\mathrm{K}_{2} \mathrm{O} / \mathrm{Na}_{2} \mathrm{O}$ & 1.8 & 1.9 & 2.6 & 1.7 & 1.8 & 2.5 & 0.6 & 1.3 & 1.0 & 0.6 & 1.2 & 1.2 & 2.2 & 3.6 \\
\hline ASI & 1.2 & 1.2 & 1.2 & 1.2 & 1.2 & 1.2 & 1.2 & 1.1 & 1.1 & 1.2 & 1.3 & 1.1 & 1.1 & 1.1 \\
\hline Cor.norm & 1.8 & 2.1 & 2.0 & 3.0 & 2.6 & 2.8 & 2.3 & 1.2 & 1.2 & 2.4 & 1.8 & 1.8 & 1.0 & 1.2 \\
\hline $\mathrm{Mg \#}$ & 49 & 47 & 47 & 50 & 49 & 49 & 44 & 44 & 45 & 46 & 46 & 45 & 40 & 36 \\
\hline $\mathrm{Rb} / \mathrm{Sr}$ & 1.9 & 2.1 & 1.0 & 1.8 & 1.9 & 0.9 & 2.0 & 2.6 & 2.3 & 2.0 & 2.5 & 2.5 & 0.9 & 1.4 \\
\hline $\mathrm{Rb} / \mathrm{Ba}$ & 0.3 & 0.3 & 0.3 & 0.3 & 0.3 & 0.2 & 0.5 & 0.5 & 0.5 & 0.5 & 0.4 & 0.5 & 0.3 & 0.7 \\
\hline $\mathrm{Rb} / \mathrm{Th}$ & 22 & 21 & 13 & & & & 8 & 9 & 7 & & & & 15 & 9 \\
\hline $\mathrm{Rb} / \mathrm{Zr}$ & 1.1 & 1.2 & 0.8 & 1.4 & 1.4 & 0.9 & 0.5 & 0.8 & 0.6 & 0.7 & 1.2 & 1.2 & 0.8 & 1.3 \\
\hline $\mathrm{Rb} / \mathrm{Hf}$ & 62 & 55 & 38 & & & & 26 & 46 & 28 & & & & 42 & 83 \\
\hline $\mathrm{Rb} / \mathrm{Y}$ & 12 & 10 & 8 & 9 & 8 & 6 & 7 & 4 & 3 & 5 & 4 & 4 & 7 & 5 \\
\hline $\mathrm{K} / \mathrm{Rb}$ & 127 & 127 & 152 & 151 & 150 & 194 & 184 & 198 & 189 & 206 & 232 & 229 & 165 & 215 \\
\hline $\mathrm{Sr} / \mathrm{Ba}$ & 0.2 & 0.1 & 0.3 & 0.2 & 0.1 & 0.3 & 0.3 & 0.2 & 0.2 & 0.3 & 0.2 & 0.2 & 0.3 & 0.5 \\
\hline $\mathrm{Th} / \mathrm{U}$ & 5 & 5 & 4 & & & & 1 & 1 & 1 & & & & 6 & 4 \\
\hline $\mathrm{Ta} / \mathrm{Nb}$ & 0.2 & 0.3 & 0.2 & & & & 0.7 & 0.7 & 0.6 & & & & 0.4 & 0.6 \\
\hline REEtotal & 253 & 284 & 284 & & & & 209 & 257 & 324 & & & & 283 & 354 \\
\hline$(\mathrm{La} / \mathrm{Sm})_{\mathrm{N}}$ & 4 & 4 & 4 & & & & 4 & 4 & 4 & & & & 4 & 3 \\
\hline$(\mathrm{La} / \mathrm{Yb})_{\mathrm{N}}$ & 13 & 9 & 11 & & & & 14 & 6 & 6 & & & & 11 & 10 \\
\hline$(\mathrm{Gd} / \mathrm{Yb})_{\mathrm{N}}$ & 2 & 2 & 1 & & & & 2 & 1 & 1 & & & & 2 & 2 \\
\hline \multirow[t]{3}{*}{$\mathrm{Eu} / \mathrm{Eu}^{*}$} & 0.6 & 0.6 & 0.6 & & & & 0.5 & 0.4 & 0.4 & & & & 0.6 & 0.3 \\
\hline & \multicolumn{5}{|l|}{ Fwifwi } & \multicolumn{3}{|c|}{ Kungwe-Kalumengongo } & \multicolumn{6}{|c|}{ Nyangwa } \\
\hline & K23.3 & K23.4 & K23.5 & K23.6 & K23.7 & K15.1 & $\mathrm{K} 15.2$ & K30 & K35.1 & K35.2 & K35.3 & K36.1 & K36.2 & K36.4 \\
\hline $\mathrm{SiO}_{2}$ & 71.38 & 70.98 & 71.81 & 69.34 & 75.74 & 69.2 & 70.26 & 70.88 & 74.05 & 76.73 & 76.53 & 68.58 & 68.01 & 67.31 \\
\hline $\mathrm{TiO}_{2}$ & 0.43 & 0.48 & 0.42 & 0.45 & 0.19 & 0.59 & 0.60 & 0.53 & 0.43 & 0.44 & 0.45 & 0.56 & 0.56 & 0.55 \\
\hline $\mathrm{Al}_{2} \mathrm{O}_{3}$ & 13.35 & 13.75 & 13.22 & 13.73 & 13.22 & 13.19 & 13.78 & 12.87 & 13.1 & 13.53 & 13.52 & 13.36 & 13.16 & 13.69 \\
\hline $\mathrm{Fe}_{2} \mathrm{O}_{3}$ & 2.38 & 2.88 & 2.07 & 2.9 & 1.54 & 3.66 & 3.67 & 3.82 & 2.13 & 2.15 & 2.15 & 4.83 & 4.79 & 4.83 \\
\hline $\mathrm{MnO}$ & 0.04 & 0.03 & 0.03 & 0.04 & 0.02 & 0.06 & 0.07 & 0.06 & 0.01 & 0.02 & 0.02 & 0.05 & 0.05 & 0.06 \\
\hline $\mathrm{MgO}$ & 0.99 & 1.01 & 0.86 & 1 & 0.44 & 1.35 & 1.47 & 1.37 & 0.72 & 0.74 & 0.74 & 2.12 & 2.18 & 2.21 \\
\hline $\mathrm{CaO}$ & 1.77 & 2.13 & 1.83 & 2.01 & 0.71 & 2.55 & 2.57 & 0.24 & 0.63 & 0.65 & 0.65 & 2.29 & 2.29 & 2.24 \\
\hline $\mathrm{Na}_{2} \mathrm{O}$ & 2.44 & 2.27 & 3.12 & 1.9 & 1.81 & 2.12 & 2.19 & 2.54 & 1.85 & 1.89 & 1.93 & 1.82 & 1.81 & 1.84 \\
\hline $\mathrm{K}_{2} \mathrm{O}$ & 5.2 & 5.13 & 3.75 & 5.9 & 6.50 & 3.82 & 3.75 & 4.89 & 5.02 & 5.03 & 5.02 & 4.09 & 4.02 & 3.94 \\
\hline
\end{tabular}




\begin{tabular}{|c|c|c|c|c|c|c|c|c|c|c|c|c|c|c|}
\hline $\mathrm{P}_{2} \mathrm{O}_{5}$ & 0.21 & 0.16 & 0.16 & 0.19 & 0.06 & 0.2 & 0.21 & 0.16 & 0.09 & 0.09 & 0.09 & 0.16 & 0.13 & 0.15 \\
\hline LOI & 1.6 & 1.27 & 1.54 & 1.68 & 1.23 & 3.29 & 2.62 & 1.89 & 1.52 & 0.48 & 0.38 & 1.55 & 2.18 & 1.45 \\
\hline Total & 99.96 & 100.2 & 98.97 & 99.33 & 101.45 & 100.1 & 101.18 & 99.42 & 99.66 & 101.74 & 101.48 & 99.53 & 99.36 & 98.25 \\
\hline Cs & 6.9 & 3.8 & 6.9 & 5.2 & & 13.3 & & 2.4 & 23.1 & & & 6.6 & 6.4 & \\
\hline $\mathrm{Rb}$ & 220 & 255 & 184.5 & 257 & 211 & 243 & 194 & 169 & 301 & 253 & 252 & 201 & 195 & 163 \\
\hline $\mathrm{Ba}$ & 1300 & 936 & 1065 & 1235 & 371 & 805 & 755 & 1345 & 587 & 594 & 588 & 896 & 887 & 870 \\
\hline Th & 12 & 16 & 13 & 14 & & 17 & & 14 & 13 & & & 14 & 14 & \\
\hline $\mathrm{U}$ & 2.5 & 2.5 & 3.5 & 3 & & 5.5 & & 6 & 3 & & & 4 & 3.5 & \\
\hline $\mathrm{Ta}$ & 4 & 6 & 5 & 12 & & 4 & & 5 & 8 & & & 7 & 12 & \\
\hline $\mathrm{Nb}$ & 12 & 15 & 13 & 16 & 12 & 16 & 14 & 15 & 15 & 12 & 12 & 15 & 16 & 13 \\
\hline $\mathrm{Sr}$ & 272 & 290 & 238 & 313 & 166 & 281 & 239 & 105 & 91 & 80 & 81 & 288 & 276 & 249 \\
\hline $\mathrm{Hf}$ & 5 & 6 & 5 & 5 & & 7 & & 6 & 5 & & & 6 & 5 & \\
\hline $\mathrm{Zr}$ & 272 & 301 & 286 & 288 & 72 & 323 & 220 & 313 & 290 & 213 & 209 & 312 & 300 & 219 \\
\hline Y & 29.5 & 35.5 & 32 & 32 & 48 & 40 & 39 & 37.5 & 35 & 38 & 35 & 33.5 & 29 & 30 \\
\hline V & 50 & 55 & 55 & 55 & 10 & 55 & 50.5 & 70 & 70 & 41.1 & 41.9 & 105 & 100 & 71.5 \\
\hline $\mathrm{Cr}$ & 46 & 44 & 44 & 42 & 4 & 64 & 61 & 42 & 30 & 37 & 30 & 82 & 84 & 84 \\
\hline $\mathrm{Ni}$ & 15 & 10 & 15 & 15 & 7 & 25 & 10 & 15 & 5 & 7 & 9 & 30 & 30 & 22 \\
\hline $\mathrm{Cu}$ & 5 & 5 & 5 & 20 & 2 & 5 & 0 & 10 & 10 & 1 & 3 & 25 & 30 & 19 \\
\hline $\mathrm{Pb}$ & 10 & 8 & 9 & 14 & 20 & 7 & 14 & 9 & 9 & 19 & 18 & 12 & 14 & 18 \\
\hline $\mathrm{Zn}$ & 30 & 20 & 35 & 90 & 26 & 50 & 31 & 45 & 25 & 17 & 16 & 70 & 65 & 48 \\
\hline Co & 86 & 69.5 & 84.5 & 137 & & 86 & & 49 & 80.5 & & & 114 & 126.5 & \\
\hline $\mathrm{La}$ & 41 & 50 & 49.5 & 43 & & 49.5 & & 52.5 & 44.5 & & & 55.5 & 54.5 & \\
\hline $\mathrm{Ce}$ & 78.5 & 101.5 & 89 & 82.5 & & 104 & & 118.5 & 86.5 & & & 108 & 106.5 & \\
\hline $\operatorname{Pr}$ & 10 & 12.5 & 11.3 & 10.2 & & 12.4 & & 12.9 & 10.1 & & & 12.5 & 12.4 & \\
\hline $\mathrm{Nd}$ & 37 & 46.5 & 41 & 38.5 & & 47 & & 47 & 35.5 & & & 45 & 45 & \\
\hline $\mathrm{Sm}$ & 7.6 & 8.7 & 7.7 & 7.2 & & 9.3 & & 9.2 & 6.7 & & & 8.1 & 8.2 & \\
\hline $\mathrm{Eu}$ & 1.3 & 1.3 & 1.4 & 1.2 & & 1.3 & & 1.4 & 0.9 & & & 1.2 & 1.3 & \\
\hline $\mathrm{Gd}$ & 5.9 & 7.8 & 6.6 & 6.4 & & 7.9 & & 7.4 & 7.3 & & & 6.9 & 6.7 & \\
\hline $\mathrm{Tb}$ & 0.9 & 1.1 & 1 & 0.9 & & 1.1 & & 1.1 & 0.9 & & & 1 & 0.9 & \\
\hline Dy & 4.9 & 6.2 & 5.4 & 5.3 & & 6.7 & & 6.8 & 5 & & & 5.3 & 5.3 & \\
\hline Ho & 1 & 1.2 & 1.1 & 1 & & 1.3 & & 1.3 & 1.1 & & & 1.2 & 1 & \\
\hline $\mathrm{Er}$ & 2.9 & 3.5 & 3 & 2.7 & & 3.8 & & 3.6 & 3.5 & & & 3.4 & 3 & \\
\hline $\mathrm{Tm}$ & 0.4 & 0.5 & 0.5 & 0.4 & & 0.6 & & 0.5 & 0.4 & & & 0.5 & 0.4 & \\
\hline $\mathrm{Yb}$ & 2.7 & 3.1 & 2.7 & 3.2 & & 3.7 & & 3.5 & 3.2 & & & 3.3 & 2.7 & \\
\hline $\mathrm{Lu}$ & 0.4 & 0.5 & 0.4 & 0.4 & & 0.6 & & 0.5 & 0.5 & & & 0.4 & 0.3 & \\
\hline $\mathrm{K}_{2} \mathrm{O} / \mathrm{Na}_{2} \mathrm{O}$ & 2.1 & 2.3 & 1.2 & 3.1 & 3.6 & 1.8 & 1.7 & 1.9 & 2.7 & 2.7 & 2.6 & 2.3 & 2.2 & 2.1 \\
\hline ASI & 1.0 & 1.1 & 1.1 & 1.0 & 1.2 & 1.1 & 1.1 & 1.3 & 1.4 & 1.4 & 1.4 & 1.5 & 1.2 & 1.2 \\
\hline Cor.norm & 0.8 & 0.8 & 1.0 & 0.9 & 2.1 & 1.3 & 2.0 & 3.2 & 3.5 & 4.0 & 3.9 & 2.0 & 1.8 & 2.7 \\
\hline Mg\# & 45 & 41 & 45 & 41 & 36 & 42 & 44 & 42 & 40 & 40 & 41 & 47 & 47 & 48 \\
\hline $\mathrm{Rb} / \mathrm{Sr}$ & 0.8 & 0.9 & 0.8 & 0.8 & 1.3 & 0.9 & 0.8 & 1.6 & 3.3 & 3.2 & 3.1 & 0.7 & 0.7 & 0.7 \\
\hline $\mathrm{Rb} / \mathrm{Ba}$ & 0.2 & 0.3 & 0.2 & 0.2 & 0.6 & 0.3 & 0.3 & 0.1 & 0.5 & 0.4 & 0.4 & 0.2 & 0.2 & 0.2 \\
\hline $\mathrm{Rb} / \mathrm{Th}$ & 18 & 16 & 14 & 18 & & 14 & & 12 & 23 & & & 14 & 14 & \\
\hline $\mathrm{Rb} / \mathrm{Zr}$ & 0.8 & 0.8 & 0.6 & 0.9 & 2.9 & 0.8 & 0.9 & 0.5 & 1.0 & 1.2 & 1.2 & 0.6 & 0.7 & 0.7 \\
\hline
\end{tabular}


Table 3 (Continued)

\begin{tabular}{|c|c|c|c|c|c|c|c|c|c|c|c|c|c|c|}
\hline & \multicolumn{14}{|l|}{ Plutons } \\
\hline & \multicolumn{5}{|l|}{ Fwifwi } & \multicolumn{3}{|c|}{ Kungwe-Kalumengongo } & \multicolumn{6}{|c|}{ Nyangwa } \\
\hline & K23.3 & K23.4 & K23.5 & K23.6 & K23.7 & K15.1 & $\mathrm{K} 15.2$ & $\mathrm{~K} 30$ & K35.1 & K35.2 & K35.3 & K36.1 & K36.2 & K 36.4 \\
\hline $\mathrm{Rb} / \mathrm{Hf}$ & 44 & 43 & 37 & 51 & & 35 & & 28 & 60 & & & 34 & 39 & \\
\hline $\mathrm{Rb} / \mathrm{Y}$ & 7 & 7 & 6 & 8 & 4 & 6 & 5 & 5 & 9 & 7 & 7 & 6 & 7 & 5 \\
\hline $\mathrm{K} / \mathrm{Rb}$ & 196 & 167 & 169 & 191 & 255 & 130 & 161 & 240 & 138 & 165 & 165 & 169 & 171 & 200 \\
\hline $\mathrm{Sr} / \mathrm{Ba}$ & 0.2 & 0.3 & 0.2 & 0.3 & 0.4 & 0.3 & 0.3 & 0.1 & 0.2 & 0.1 & 0.1 & 0.3 & 0.3 & 0.3 \\
\hline $\mathrm{Th} / \mathrm{U}$ & 5 & 6 & 4 & 5 & & 3 & & 2 & 4 & & & 4 & 4 & \\
\hline $\mathrm{Ta} / \mathrm{Nb}$ & 0.3 & 0.4 & 0.4 & 0.8 & & 0.3 & & 0.3 & 0.5 & & & 0.5 & 0.7 & \\
\hline REEtotal & 224 & 280 & 253 & 235 & & 289 & & 304 & 241 & & & 286 & 277 & \\
\hline$(\mathrm{La} / \mathrm{Sm})_{\mathrm{N}}$ & 3 & 4 & 4 & 2 & & 3 & & 4 & 4 & & & 4 & 4 & \\
\hline$(\mathrm{La} / \mathrm{Yb})_{\mathrm{N}}$ & 10 & 11 & 12 & 9 & & 9 & & 10 & 9 & & & 11 & 14 & \\
\hline$(\mathrm{Gd} / \mathrm{Yb})_{\mathrm{N}}$ & 2 & 2 & 2 & 2 & & 2 & & 2 & 2 & & & 2 & 2 & \\
\hline $\mathrm{Eu} / \mathrm{Eu}^{*}$ & 0.6 & 0.5 & 0.6 & 0.4 & & 0.5 & & 0.5 & 0.4 & & & 0.5 & 0.5 & \\
\hline
\end{tabular}

Samples with REE contents provided were analysed using ICP at Chemex Laboratory (Canada). The other samples were analysed using X-ray fluorescence (XRF) at Osaka City University (Japan). 

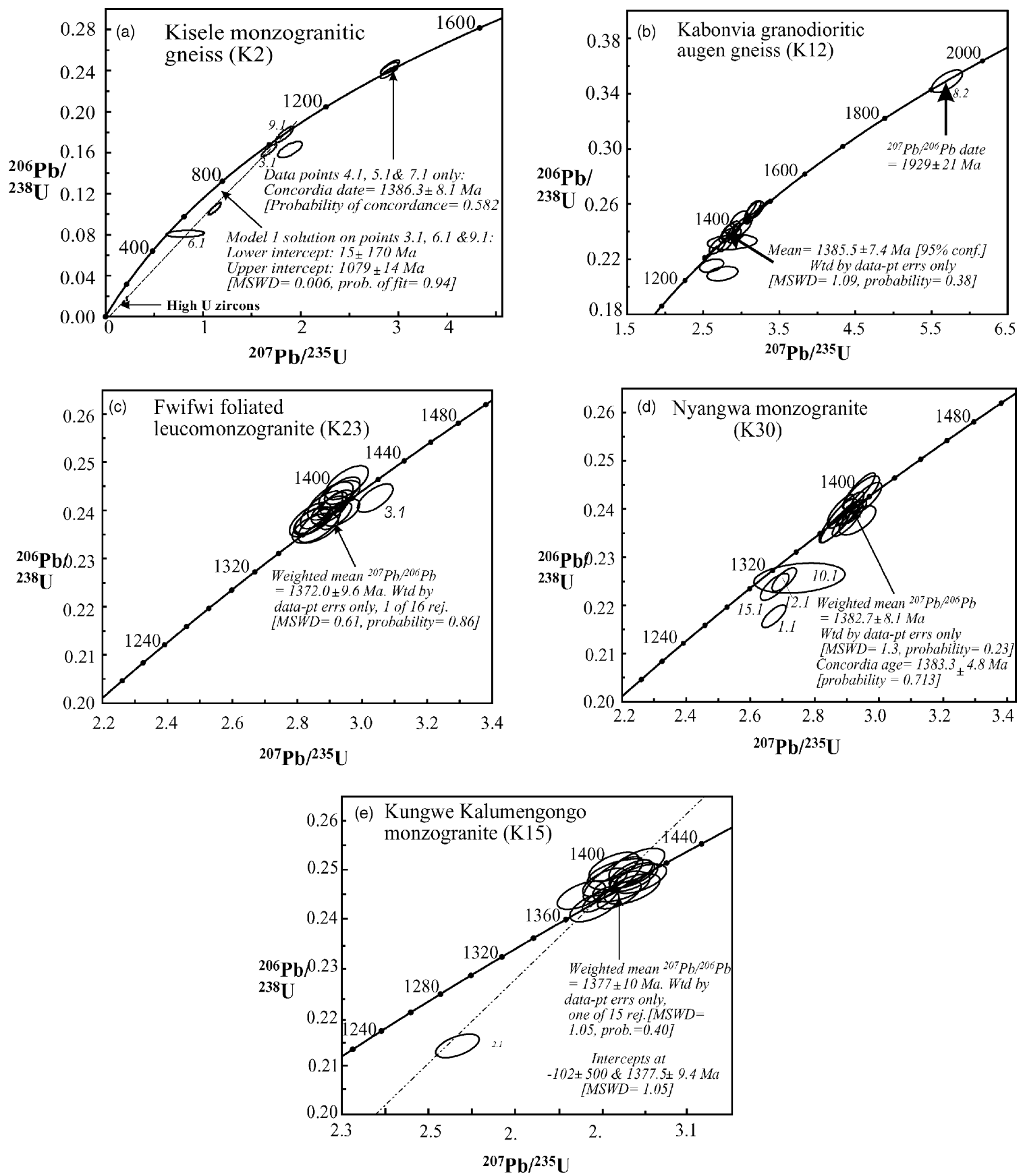

Fig. 5. Wetherill U-Pb concordia diagrams for: (a) Kisele monzogranitic gneiss (K2). The dashed line represents the regression of the data for the three low Th/U (metamorphic) analyses; (b) Kabonvia granodioritic augen gneiss (K12); (c) Fwifwi foliated leucomonzogranite (K23). The age shown is calculated from the dotted error ellipses; (d) Kungwe-Kalumengongo stock (K15). The black error ellipse represents the data for analysis 2.1 , which was not included in the age calculation for this sample; (e) Nyangwa monzogranite (K30). The discordant data represented by the dotted error ellipses were not included in the age calculations. For all these diagrams, the data-point error ellipses are plotted with $1 \sigma$ errors. 
discordant, having clearly suffered severe Pb-loss as a consequence of high and sometimes extreme $U$ content (e.g. analysis 10.1 has an $\mathrm{U}$ concentration of over 1.1 wt.\%). For these analyses, cores were avoided, and most analyses were sited in the zoned magmatic area of the grains. Three such analyses (4.1, 5.1 and 7.1) yielded data within error of concordia and these can be combined to give a Concordia age of $1386.3 \pm 8.1 \mathrm{Ma}$ (probability of concordance $=0.582$ ). This date is interpreted to represent the best estimate of the age of emplacement of the protolith of this granite.

Three analyses (3.1, 6.1 and 9.1) have unusually low $\mathrm{Th} / \mathrm{U}$ composition compared to the remainder of the population. Two of these analyses were sited on the rims of uniform zircon and the third (9.1) in a uniform zone, i.e. without the concentric compositional zoning seen in the magmatic population. These three data points range from 1 to $53 \%$ discordant and lie on a discordia for which an upper intercept age of $1079 \pm 14 \mathrm{Ma}$ can be calculated. The low $\mathrm{Th} / \mathrm{U}$ values in these zircon overgrowths are commonly observed in metamorphic zircons and thus these three analyses are interpreted to date a metamorphic event that affected this granite, and the zircons within it.

\subsection{Kabonvia granodioritic augen gneiss}

The zircons from this sample are euhedral to subhedral, with clear elongate grains mixed with larger, oblate and somewhat chunky, brown crystals. Cathodoluminescence revealed numerous cores of variable dimensions, with the general population comprising strongly zoned (concentric compositional zoning) zircons.

Sixteen $\mathrm{U}-\mathrm{Th}-\mathrm{Pb}$ analyses were done on 15 different zircons from this ortho gneiss. The data are listed in Table 2 and are plotted on a conventional Wetherill U-Pb concordia plot (Fig. 5b). Most of the analyses plot as a group near the concordia, for which a weighted mean ${ }^{207} \mathrm{~Pb} /{ }^{206} \mathrm{~Pb}$ date of $1385.5 \pm 7.4 \mathrm{Ma}$ $(\mathrm{MSWD}=1.09 ; P=0.36)$ can be calculated. Two analyses (4.1 and 13.1), which were excluded from the above age calculation, are significantly discordant and have relatively high common $\mathrm{Pb}$ content. A single large, sector-zoned core (8.2) was analysed and yielded a concordant ${ }^{207} \mathrm{~Pb} /{ }^{206} \mathrm{~Pb}$ crystallisation age of $1929 \pm 21 \mathrm{Ma}$. The zoned, magmatic overgrowth on this xenocrystic zircon formed during the crystallisa- tion of the gneiss protolith, giving a ${ }^{207} \mathrm{~Pb} /{ }^{206} \mathrm{~Pb}$ age of $1398 \pm 13 \mathrm{Ma}(1 \sigma)$, an age indistinguishable from the magmatic age calculated above.

\subsection{Fwifwi foliated leucomonzogranite}

Zircons from this sample are euhedral to subhedral, and light pink to brown. The general population comprises strongly zoned zircon, consistent with crystallisation from felsic magma. Many grains have cores of inherited zircon. Sixteen U-Th-Pb analyses were done on different grains from this granite, targeting the zoned magmatic area of the grains. The results are shown in Table 2 and plotted in Fig. 5c. All analyses are very uniform with respect to $\mathrm{Th} / \mathrm{U}$ with an average value of 0.32 , and apart from one analysis (3.2), the data form a group around concordia. Fifteen analyses can be combined to give a weight mean ${ }^{207} \mathrm{~Pb} /{ }^{206} \mathrm{~Pb}$ date of $1372 \pm 9.6 \mathrm{Ma}(\mathrm{MSWD}=0.61 ; P=0.86)$.

\subsection{Nyangwa monzogranite}

The zircons from this sample are very variable in size and shape, and are generally brown in colour. Some of the grains are fragments of presumably once larger grains. The zoning patterns vary between areas of weak, broad zonation and areas of rapidly changing concentric compositional zoning. Core of inherited fragments of various sizes are preserved.

Most of the 16 analyses performed on the zircon from this sample (Table 2) plot as a cluster on concordia (Fig. 5d), and gives a weighted mean ${ }^{207} \mathrm{~Pb} /{ }^{206} \mathrm{~Pb}$ age of $1382 \pm 8.1 \mathrm{Ma}(\mathrm{MSWD}=1.3 ; P=0.23$ ). The age from the same data set can be further constrained by the calculation of the Concordia age of $1383.3 \pm 4.8 \mathrm{Ma}$ (probability of concordance $=0.713$ ). Four discordant analyses (1.1, 10.1, 12,1 and 15.1) were excluded from all age calculations. The concordia date of $1383.3 \pm 4.8 \mathrm{Ma}$ is the best estimate of the age of crystallisation of this pluton.

\subsection{Kungwe-Kalumengongo monzogranite}

This sample produced a good crop of excellent quality euhedral and subhedral zircons. The zircons are clear, transparent and light pink. Only a few, small apparent core (not analysed) are observed through the use of cathodoluminescence imaging, with most 
zircons comprising simple concentric compositional zoning.

Fifteen different zircons were analysed from this sample. The results are plotted in Fig. 5e and are listed in Table 2. The zircons appear to be simple magmatic grains with very uniform $\mathrm{U}, \mathrm{Th}$ and $\mathrm{Th} / \mathrm{U}$ contents. Apart from a single discordant analysis (2.1), the data plot as a group on or near concordia. A weighted mean ${ }^{207} \mathrm{~Pb} /{ }^{206} \mathrm{~Pb}$ date of $1377 \pm 10 \mathrm{Ma}(\mathrm{MSWD}=1.05$; $P=0.40)$ calculated from this group of analyses is interpreted to be the magmatic age of this granite.

\section{Whole-rock geochemistry}

Representative whole-rock analyses are listed in Table 3. The composition of the Mitwaba granitoids falls between 67.24 and 76.73 wt. $\% \mathrm{SiO}_{2}$. All the rocks are potassic, with $\mathrm{K}_{2} \mathrm{O} / \mathrm{Na}_{2} \mathrm{O}>1$, except for sample $\mathrm{K} 2.1$ in which $\mathrm{K}_{2} \mathrm{O}$ loss is suspected. In the Qz-Ab-Or normative diagram (Fig. 6a), the Mitwaba granitoids show a near eutectic minimum-temperature melt composition and they overlap with the field of experimental felsic melts originating from dehydration melting of metasedimentary rocks (e.g. Stevens et al., 1997). All Mitwaba granitoids have normative corundum in the range $0.8-4.8$ and its content is not correlated to loss-on-ignition values. The alumina saturation index (ASI: molar $\mathrm{Al}_{2} \mathrm{O}_{3} / \mathrm{CaO}+\mathrm{Na}_{2} \mathrm{O}+\mathrm{K}_{2} \mathrm{O}$ ) is in the range 1.1-1.5, indicating strongly peraluminous compositions (Fig. 6b). These granitoids have low total $\mathrm{Fe}_{2} \mathrm{O}_{3}, \mathrm{Na}_{2} \mathrm{O}, \mathrm{MgO}$ and $\mathrm{CaO}$ (Table 3) and are characterized by: (1) $\mathrm{CaO} / \mathrm{Na}_{2} \mathrm{O}$ in the range $0.3-1.2$, except in the Kisele pluton where lower values occur; and (2) $\mathrm{Al}_{2} \mathrm{O}_{3} / \mathrm{Na}_{2} \mathrm{O}$ between 22 and 31, except in the Kisele pluton marked by higher ratios (71:81). The composition and inter-oxide ratios of major elements show that Mitwaba strongly peraluminous (SP) granitoids are similar to Hercynian and Lachlan Fold belts SP granitoids (e.g. Fig. 6c and d) and have characteristics of synorogenic anatectic SP granitoids originating from partial melting of metasedimentary rocks (Fig. 6d and e). Mg\# versus $\mathrm{CaO}$ diagram (Fig. 6f) indicates distinct evolution trends between and within plutons from the Mitwaba SP granitoids.

$\mathrm{Rb}$ and $\mathrm{Sr}$ contents range between 119-329 and 61-313 ppm, respectively. $\mathrm{K} / \mathrm{Rb}$ ratios are in the range 150-260, except in two samples marked by lower ratios close to $130 . \mathrm{K} / \mathrm{Rb}>150$ usually mark granitoids not affected by post-magmatic activity of aqueous phase. Ba concentrations are between 229 and $1000 \mathrm{ppm}$, except in three samples containing $1100-1400 \mathrm{ppm} \mathrm{Ba}$. $\mathrm{Rb} / \mathrm{Ba}$ ratios are in the range $0.2-0.7$, excluding the three samples with high $\mathrm{Ba}$ content leading to lower $\mathrm{Rb} / \mathrm{Ba}<0.2 \mathrm{Rb} / \mathrm{Sr}$ is in the range 0.7-3.3 whereas $\mathrm{Sr} / \mathrm{Ba}$ ratios are between 0.1 and 0.3 in Mitwaba granitoids. With a few exceptions, the above trace element contents compare with values reported for S-type granitoids. Indeed, granitoids resulting from dehydration melting of a metapelitic precursor are characterized by $\mathrm{Rb}>100 \mathrm{ppm}, \mathrm{Sr}<$ 300-400 ppm, $\mathrm{Ba}<600-1000 \mathrm{ppm}$ and $\mathrm{Rb} / \mathrm{Ba}$ ratios $>0.25$ (Miller, 1985).

$\mathrm{Zr}$ shows wide scatter and is not correlated with $\mathrm{SiO}_{2}$. Its concentration is medium to high, frequently between 131 and $323 \mathrm{ppm}$ (except in sample K23.4 containing $72 \mathrm{ppm})$. S-type granites are commonly characterized by low $\mathrm{Zr}$ content $(<100 \mathrm{ppm}$; e.g. Scaillet et al., 1990) because of the low solubility of zircon in peraluminous melts formed at relatively low $\left(<800^{\circ} \mathrm{C}\right)$ temperature (Watson and Harrison, 1983). However, large volume SP granitoids generated at higher temperatures $\left(>875^{\circ} \mathrm{C}\right)$ as documented in the Lachlan and Hercynian belts display higher $\mathrm{Zr}$ contents, similar to the high $\mathrm{Zr}$ values recorded in the Mitwaba SP granitoids. Rb/Zr ratios in Mitwaba granitoids are between 0.5 and 1.4 and this ratio is usually $>1$ in Himalaya-type S-type leucogranites (e.g. Harris and Inger, 1992) and $<1$ in Lachlan-type SP granitoids. In the diagrams using $\mathrm{Nb}, \mathrm{Y}$ and $\mathrm{Rb}$, the Mitwaba granitoids cluster at the boundary between within-plate, collisional and volcanic-arc granitoids (Fig. 7) and specifically overlap with the field for post-collisional granitoids of Pearce (1996). We are aware that the position of the data in these diagrams precludes their use for tectonic setting interpretation but we are more interested by showing that again the Mitwaba granitoids overlap the average composition of the Lachlan SP granitoids. These granitoids are characterized by $\mathrm{Nb}$ content between 6 and $16 \mathrm{ppm}$ and Ta concentrations between $\sim 3$ and $12 \mathrm{ppm}$, yielding $\mathrm{Ta} / \mathrm{Nb}$ ratios in the range $0.2-0.8$. For the above range of $\mathrm{Nb}$, granitoids containing Ta contents higher than $2 \mathrm{ppm}$ are commonly of S-type affinity (Harris et al., 1986). 

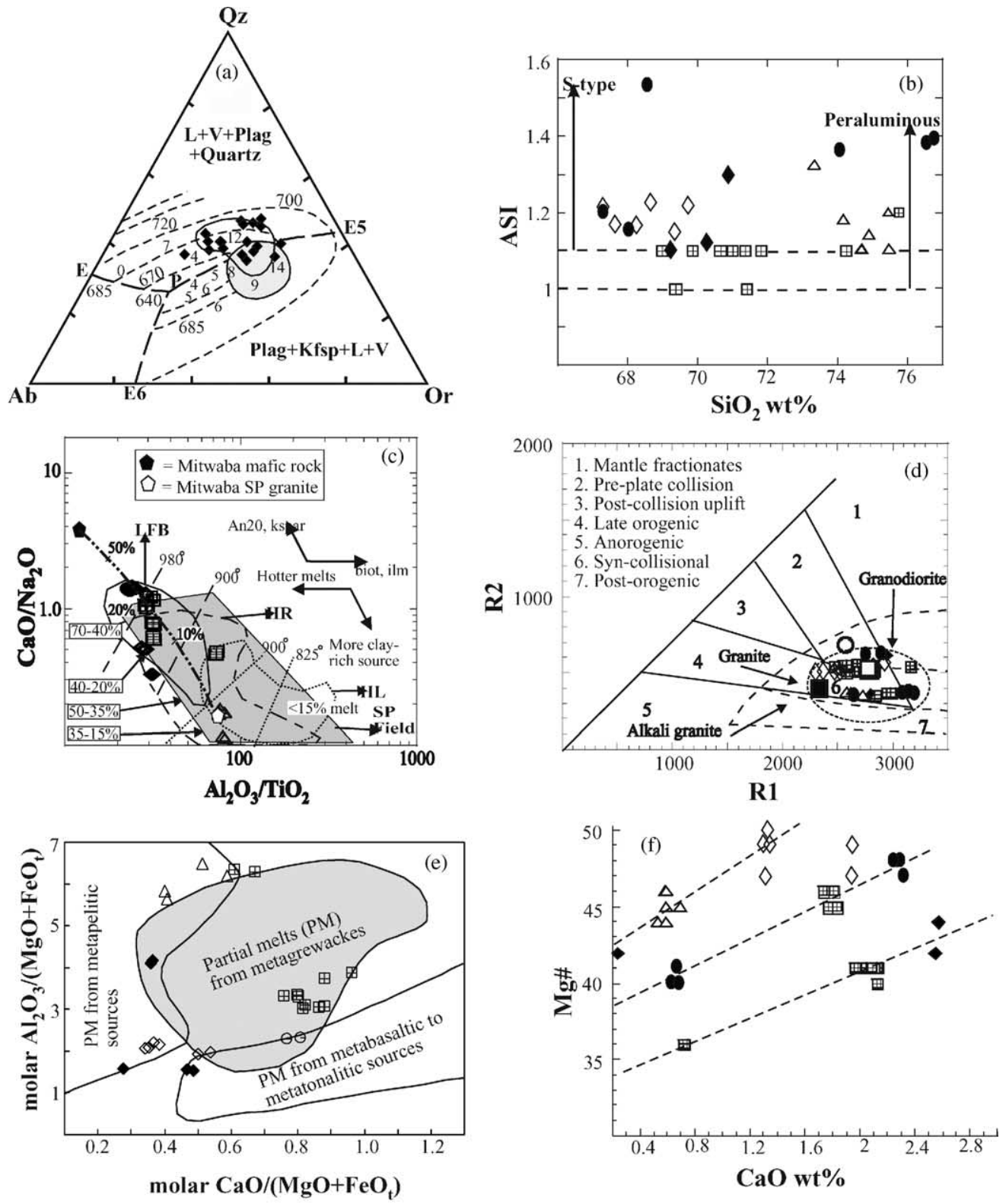

\section{Symbols:}

$\diamond$ Kabonvia granodioritic augen gneiss

$\triangle$ Kisele monzogranitic gneiss

$\boxplus$ Fwifwi foliated leucomonzogranite

- Nyangwa monzogranite

LFB: Field for Lachlan Fold Belt SP

HL: Field for Himalayan belt SP

Kungwe-Kalumengongo monzogranite

- Average Himalayan leucogranites

O Average I-type granites from LFB

口 Average S-type granites from LFB

HR: Field for Hercynian belt SP

SP: Strongly Peraluminous Granites 

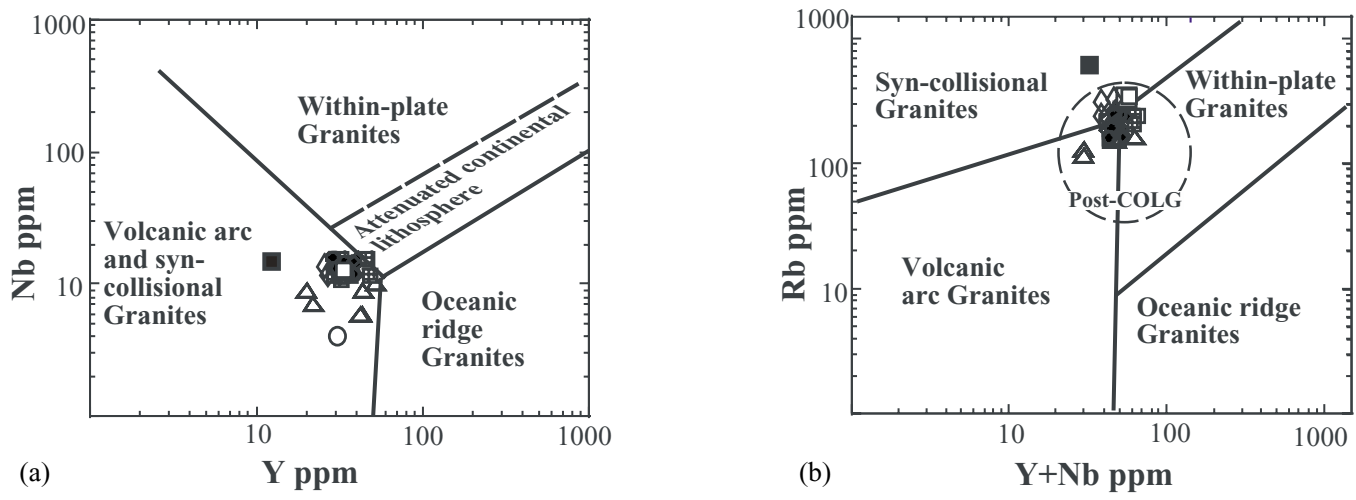

\begin{tabular}{|ll|}
\hline Symbols: & \\
$\diamond$ Kabonvia granodioritic augen gneiss & $\diamond$ Kungwe-Kalumengongo monzogranite \\
$\Delta$ Kisele monzogranitic gneiss & average Himalayan leucogranites \\
田 Fwifwi foliated leucomonzogranite & $\bigcirc$ Average I-type granites from Lachlan Fold Belt (LFB) \\
$\bullet$ Nyangwa monzogranite & $\square$ Average S-type granites from LFB \\
\hline
\end{tabular}

Fig. 7. (a) Nb vs. $\mathrm{Y}$; and (b) Rb vs. (Y + Nb) tectonic setting discrimination diagrams of Pearce et al. (1984) and Pearce (1996). See the text for more details.

Th contents vary between 13 and $25 \mathrm{ppm}$, except in one sample containing $27 \mathrm{ppm}$ Th. Uranium ranges between 2.5 and $7.5 \mathrm{ppm}$, except in three samples containing between 13 and $19 \mathrm{ppm}$. The $\mathrm{Th} / \mathrm{U}$ ratio is variable and range from 1 to 6 , with an average of 3.7 , which is close to the crustal average of 3.8 (Taylor and McLennan, 1985). S-type felsic magmas derived from the melting of sedimentary rocks are usually characterized by $\mathrm{Th}<25 \mathrm{ppm}$ and $\mathrm{U}<10 \mathrm{ppm}$ (e.g. Taylor and McLennan, 1985). $\mathrm{Rb} / \mathrm{Th}$ ratios in Mitwaba granitoids are higher than the chondritic value $(\sim 8)$. They range generally from 13 to 23 , with five samples showing lower values $(\sim 8-12)$.
The rare earth elements (REE) patterns are illustrated in Fig. 8a and b. The Mitwaba granitoids are enriched in REE and display moderately to strongly fractionated patterns $\left(\mathrm{La}_{\mathrm{N}}=59-84, \mathrm{Yb}_{\mathrm{N}}=\right.$ $\left.4-12, \mathrm{La}_{\mathrm{N}} / \mathrm{Yb}_{\mathrm{N}} \sim 6-14\right)$. The REE fractionation is greater for LREE $\left(\mathrm{La}_{\mathrm{N}} / \mathrm{Sm}_{\mathrm{N}}=2-4\right)$ than for HREE $\left(\mathrm{Gd}_{\mathrm{N}} / \mathrm{Yb}_{\mathrm{N}}=1-2\right)$. The Kisele monzogranite is mainly characterized by sub-flat HREE patterns (Fig. 8b). LREE and HREE are not correlated with silica content and a negative europium anomaly occurs in all the samples $\left(\mathrm{Eu} / \mathrm{Eu}^{*}=0.3-0.6\right)$. For comparison, S-type leucogranites are commonly characterized by $\mathrm{La}_{\mathrm{N}}<100, \mathrm{Yb}_{\mathrm{N}}<10$ and are marked by strong

Fig. 6. (a) Normative Qz-Ab-Or diagram (part of the Qz-Ab-Or-An- $\mathrm{H}_{2} \mathrm{O}$ system) showing the low-temperature melt area near the cotectic region (E5-P) at $5 \mathrm{~kb}$ (Winkler et al., 1975). Small numbers give anorthite contents at the intersection of the isotherms (dashed lines) with the cotectic planes. Most of the Mitwaba granitoid samples (black diamonds) plot in the minimum melt low-temperature region of this system. Also shown in this diagram are fields of felsic melts originating from experimental dehydration melting of metasedimentary rocks at $850^{\circ} \mathrm{C}$ and $0.5 \mathrm{GPa}$ (open field) and at $900^{\circ} \mathrm{C}$ and $1 \mathrm{GPa}$ (grey-shaded field) (Stevens et al., 1997). See text for details. (b) Plot of $\mathrm{SiO}_{2}$ against ASI [molar $\mathrm{Al}_{2} \mathrm{O}_{3} /\left(\mathrm{CaO}+\mathrm{Na}_{2} \mathrm{O}+\mathrm{K}_{2} \mathrm{O}\right)$ ] for Mitwaba granitoids. (c) $\mathrm{CaO} / \mathrm{Na}_{2} \mathrm{O}$ vs. $\mathrm{Al}_{2} \mathrm{O}_{3} / \mathrm{TiO}_{2}$ ratios for experimental vapour-absent melts from natural rocks (data from Sylvester, 1998). Temperatures and percentage of melt produced are shown. The mixing curve (dashed-dotted line), with percentage of mixing between end members of Mitwaba mafic and felsic igneous rocks is shown in comparison to the field for strongly peraluminous granites. Fields for Himalayan (HL), Hercynian (HR) and Lachlan Fold belt (LFB) SP granitoids are drawn. Vectors indicate that $\mathrm{Al}_{2} \mathrm{O}_{3} / \mathrm{TiO}_{2}$ ratios decrease with the increase of melt temperature, whereas $\mathrm{CaO} / \mathrm{Na}_{2} \mathrm{O}$ ratios decrease with the increase of clay-rich source-rocks. See the text for discussion. (d) R1 and R2 chemical parameters (De La Roche et al., 1980). $\mathrm{R} 1=4 \mathrm{Si}-11(\mathrm{Na}+\mathrm{K})-2(\mathrm{Fe}+\mathrm{Ti}) ; \mathrm{R} 2=6 \mathrm{Ca}+2 \mathrm{Mg}+\mathrm{Al}$. Also shown in this diagram is the field of syn-orogenic granitoids originating from partial melting of crustal rocks (Batchelor and Bowden, 1985). (e) $\mathrm{Molar} \mathrm{Al}_{2} \mathrm{O}_{3} /(\mathrm{MgO}+\mathrm{FeO} t) \mathrm{vs} . \mathrm{CaO} /(\mathrm{MgO}+\mathrm{FeO} t)$ for Mitwaba granitoids. The fields shown are from Altherr et al. (2000). See the text for further details. (f) $\mathrm{Mg \#} \mathrm{vs.} \mathrm{CaO} \mathrm{diagram} \mathrm{for}$ Mitwaba granitoids (see the text for details). 

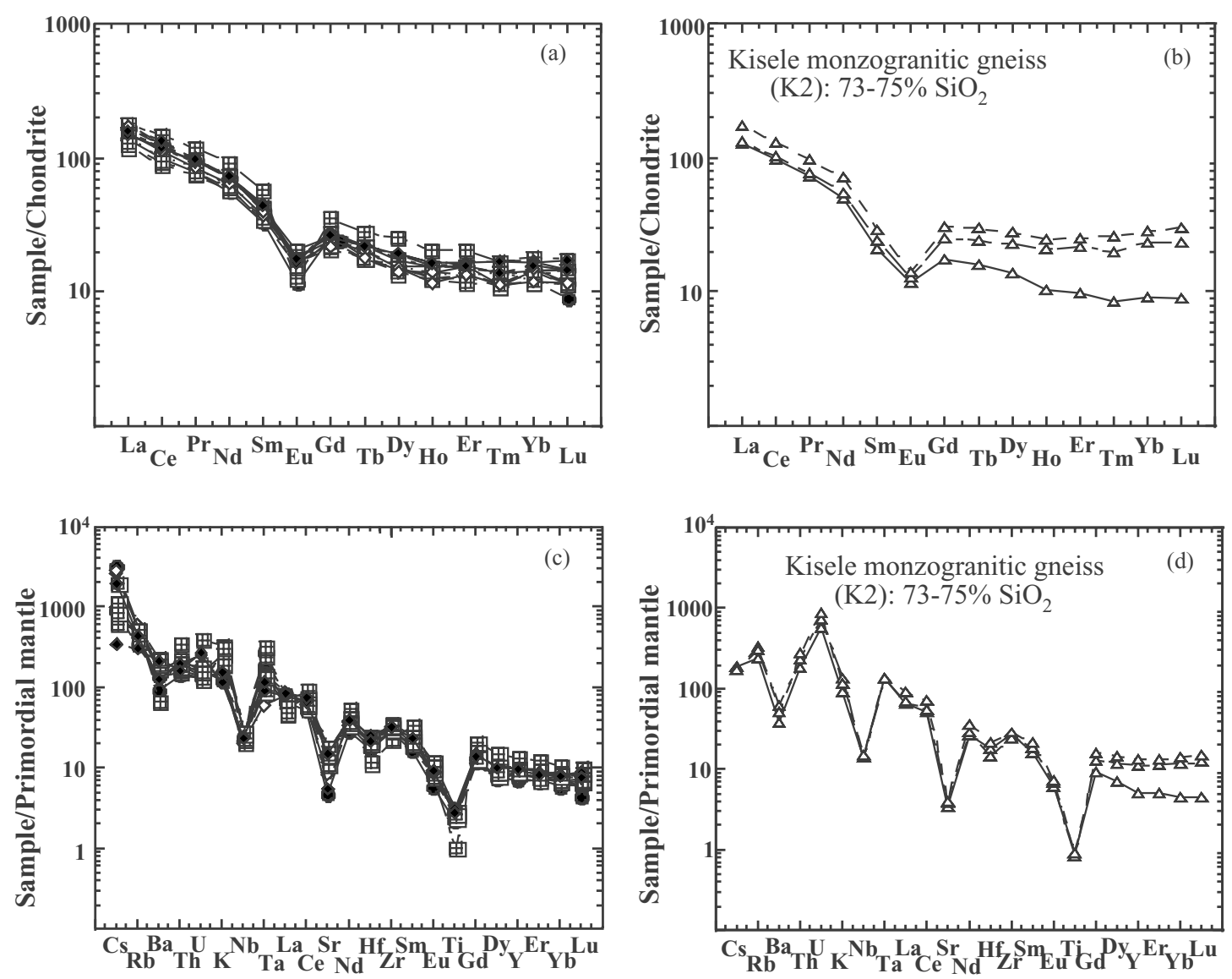

For (a) and (c)

$\diamond$ Kabonvia granodioritic augen gneiss (K12): 67-69\% $\mathrm{SiO} 2$

田 Fwifwi foliated leucomonzogranite (K23): 69-76\% SiO2

- Kungwe-Kalumengongo monzogranite (K15): 69-71\% SiO2

- Nyangwa monzogranite (K30): 67-77\% SiO2

Fig. 8. ( $a$ and b) Chondrite-normalized REE patterns; and ( $c$ and d) primordial mantle-normalized spider diagrams for Mitwaba granitoids. Normalizing values of Sun and McDonough (1989).

negative $\mathrm{Eu}$ anomaly shown by $\mathrm{Eu} / \mathrm{Eu}^{*}<0.5$ (e.g. Williamson et al., 1996). Zr content shows a good correlation with HREE as illustrated in $\mathrm{Zr}$ versus $\mathrm{Yb}$ diagram (Fig. 9), whereas there is no obvious correlation with LREE such as La.

Primordial mantle-normalized diagrams (Fig. 8c and d) show marked negative anomalies in $\mathrm{Ti}, \mathrm{Sr}, \mathrm{Nb}$ and subsidiary $\mathrm{Ba}$. The Kisele monzogranite gneiss exhibits a positive $U$ anomaly. This whole-rock uranium anomaly correlates with high $\mathrm{U}$ content in zircons from this pluton (see Section 6).

\section{Discussion}

\subsection{Geochronological results}

The following are the igneous crystallisation ages for the Mitwaba granitoids: $1386.3 \pm 8.1 \mathrm{Ma}$ (Kisele monzogranite gneiss), $1385.5 \pm 7.4 \mathrm{Ma}$ (Kabonvia granodioritic augen gneiss), $1383.3 \pm 4.8 \mathrm{Ma}$ (Nyangwa monzogranite), $1377 \pm 10 \mathrm{Ma}$ (KungweKalumengongo monzogranite) and $1372 \pm 9.6 \mathrm{Ma}$ (Fwifwi foliated leucomonzogranite). The dated gran- 


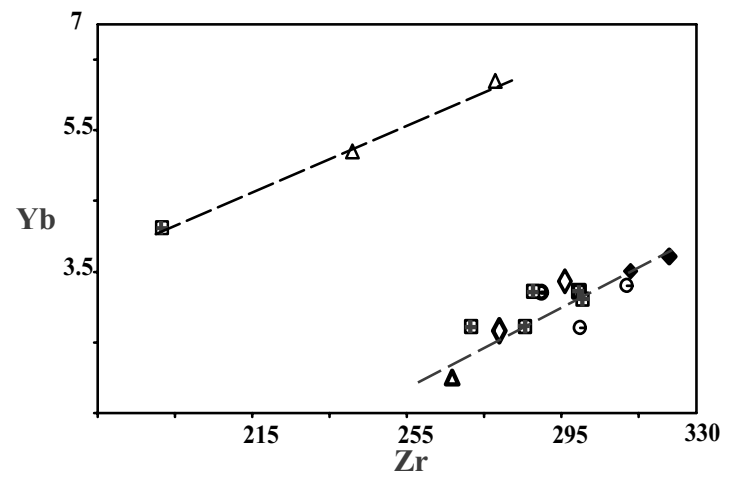

Fig. 9. Zr vs. Yb plot for Mitwaba granitoids. Symbols: see Fig. 7.

itoids cover the spectrum from $\mathrm{G}_{1}$ (type A) Kibaran granitoids previously inferred to be emplaced at $1331 \pm 25$ Ma up to $G_{2}$ (type B) granitoids supposedly intruded at $1308 \pm 25 \mathrm{Ma}$ and $\mathrm{G}_{3}$ (type $\mathrm{C}$ ) granitoids assumed to be younger and emplaced at around $1094 \pm 50$ Ma (e.g. Cahen et al., 1984; Klerkx et al., 1987; Fernandez-Alonso et al., 1986). It emerges from this SHRIMP U-Pb zircon geochronological study that all these granitoids were emplaced over a short period of time, between $1386.3 \pm 8.1 \mathrm{Ma}$ and $1372 \pm 9.6 \mathrm{Ma}$, and considering the uncertainty on the individual dates, all these plutons could be coeval at $1381 \pm 8 \mathrm{Ma}$. These geochronological data support the conclusion of Kampunzu et al. (1986) who indicated, based on structural data, that the so-called types A-B-C $\left(\mathrm{G}_{1}-\mathrm{G}_{2}-\mathrm{G}_{3}\right)$ Kibaran granitoids were early syn-kinematic $\mathrm{D}_{1}$ Kibaran intrusive bodies. The age of these granitoids date the first contractional deformation in the Kibaran, at least in the Mitwaba area.

The age of the second Kibaran deformation event is younger than $1381 \pm 8 \mathrm{Ma}$, taken above to be the age of $\mathrm{D}_{1}$, and is known to be older than the tin granite emplaced between ca. 1000 and $950 \mathrm{Ma}$ (Cahen et al., 1984; Ikingura et al., 1992; Romer and Lehmann, 1995). The Kisele monzogranite gneiss contains metamorphic zircon overgrowths with low $\mathrm{Th} / \mathrm{U}$ ratios and dated at $1079 \pm 14 \mathrm{Ma}$. Because the Kisele foliated monzogranite is deformed, developing gneissosity with a strong NE-trending solid-state $S_{2}$ fabric marking the Kibaran paroxysmal compressional deformation $\left(\mathrm{D}_{2}\right)$ superimposed on an earlier fabric reported to $\mathrm{D}_{1}$, we tentatively conclude that the metamorphic age of $1079 \pm 14$ Ma dates the second Kibaran oro- genic event $\left(\mathrm{D}_{2}\right)$. Additional geochronological data are required to constrain this interpretation. Metamorphic zircon overgrowth was recorded only in the Kisele pluton and is lacking in all other granitoids investigated. This difference probably reflects the fact that the Kisele pluton is the only one among the studied granitoid bodies that was reworked under sillimanite zone metamorphism (Table 1), i.e. under temperatures high enough for metamorphic zircon overgrowth. All other plutons were reworked under chlorite zone (Nyangwa, Kungwe-Kalumengongo) or staurolite zone (Kabonvia) metamorphism, i.e. below the temperature required for metamorphic zircon overgrowth (e.g. Mezger and Krogstad, 1997). The large number of low-precision $\mathrm{Rb}-\mathrm{Sr}$ ages of $1094 \pm 50 \mathrm{Ma}$ reported by previous workers as the emplacement age for $\mathrm{G}_{3}$ granitoids is, within the margin of errors, similar to the metamorphic zircon age reported above. In our interpretation, these old $\mathrm{Rb} / \mathrm{Sr}$ $\mathrm{G}_{3}$ Kibaran granitoid dates from the Kibaran belt in central and eastern Africa record the resetting of the $\mathrm{Rb}-\mathrm{Sr}$ systematic during the $\mathrm{D}_{2}$ deformation event.

This study also indicates that all the studied granitoids contain inherited zircons, one of them yielding a Paleoproterozoic age of $1929 \pm 21 \mathrm{Ma}$. There is no field evidence to suggest that the Mitwaba granitoids were emplaced through an older basement. Thus, we infer that the "old" zircons in these granitoids are inherited from the metasedimentary source of the granitic magma.

\subsection{Characterization of the Mitwaba granitoids}

The Mitwaba granitoids have the following characteristics:

(1) In the field, they define very large batholiths (e.g. Kifinga-Kisele batholith: $70 \mathrm{~km} \times 65 \mathrm{~km}$ ) surrounded by small satellite plutons. Apart from the Kisele foliated monzogranite gneiss, most of these granitoids intrude into low-to-medium grade metasedimentary rocks. Large batholiths such as Kifinga-Kisele are rimmed by contact metamorphic aureoles (garnet-hornblende hornfels). The granitoids contain restitic micas-rich clots as well as enclaves of metasedimentary rocks. These observations are similar to those described for the Lachlan and Hercynian belts 
SP granitoids originating from partial melting of a metasedimentary precursor (e.g. Chappell and White, 1992; Sylvester, 1998).

(2) The Mitwaba granitoids are devoid of hornblende and pyroxene and contain biotite and primary muscovite. Biotites are Al-rich and have $\mathrm{Fe}-\mathrm{Mg}-\mathrm{Al}$ relationship suggesting that they grew in peraluminous melts. The mineralogical composition of the Mitwaba granitoids shows that they belong to muscovite-bearing peraluminous group of Barbarin (1999). Monazite is a common accessory mineral, tourmaline occurs in several samples and these rocks are similar to the "ilmenite series" granitoids of Ishihara (1977).

(3) Whole rock chemical compositions of these granitoids are characterized by high silica content (67.24-76.73wt.\%), ASI values between 1.04 and 1.6 (Fig. 6b) and up to $4.8 \%$ corundum in the normative composition. All the granitoids are potassic. $\mathrm{Rb}, \mathrm{Sr}, \mathrm{Ba}$ concentrations and ratios of trace elements such as $\mathrm{Rb} / \mathrm{Th}$ and $\mathrm{Rb} / \mathrm{Ba}$ suggest an S-type nature for the Mitwaba granitoids. For example, $\mathrm{Rh} / \mathrm{Th}$ values higher than 20 , low $\mathrm{Th} / \mathrm{U}$ ratios (average 3.6), high Ta contents and high $\mathrm{Rb} / \mathrm{Hf}$ (26-83), Rb/Y (3.2-12) and $\mathrm{Ta} / \mathrm{Nb}$ $(0.19-1.15)$ in these granitoids suggest that they are S-type felsic melts derived from a sedimentary protolith (e.g. Harris and Inger, 1992).

(4) Primordial mantle normalized patterns (Fig. 8) exhibits $\mathrm{Ba}, \mathrm{Nb}, \mathrm{Sr}$ and $\mathrm{Ti}$ negative anomalies. The high field strength element (HFSE) anomalies (mainly $\mathrm{Nb}$ and $\mathrm{Ti}$ ) are known to be characteristic of subduction zones (e.g. Saunders et al., 1991) and igneous rocks originating from the continental crust (e.g. Tarney and Jones, 1994). For S-type granitoids, it has been suggested that the $\mathrm{Sr}$ negative anomaly coupled to low $\mathrm{Na}_{2} \mathrm{O}$, $\mathrm{CaO}$ and high $\mathrm{K}_{2} \mathrm{O}$ contents reflect the low versus high concentration of these elements in the source, most probably weathered metasedimentary rocks (e.g. Chappell and White, 1992). The LREE enrichment (e.g. $\mathrm{La}_{\mathrm{N}}=59-84$ times chondrite) relative to HREE (e.g. $\mathrm{Yb}_{\mathrm{N}}=4-12$ times chondrite) results most probably from restitic zircon (Harris and Inger, 1992) and this is supported by the correlation between $\mathrm{Zr}$ and HREE (e.g. Fig. 9). The composition of Mitwaba granitoids (e.g. Fig. 6) overlaps the field of fel- sic melts originating from dehydration melting of metapelites/metagreywackes precursors (e.g. Stevens et al., 1997). The only potential metasedimentary source rocks for these granitoids are the Mitwaba Group supracrustal metasedimentary rocks. The Mulumbi group sedimentary rocks post-date the emplacement of these granitoids.

(5) Cathodoluminescence and SHRIMP data indicate that igneous zircons in these granitoids contain nearly rounded inherited zircon cores, although in a few samples, cores of zircon show irregular shapes. As stressed above, there is no field evidence suggesting that these granitoids were emplaced through an older basement and thus we infer that the xenocrystic zircons were inherited from the metasedimentary source of the magma. The rounding of most of the "old" zircon cores could be of metamorphic origin (e.g. Mezger and Krogstad, 1997), although it could as well result partly from pre-metamorphic sedimentary rounding (e.g. Williams, 2001).

These petrographic and geochemical characteristics of the Mitwaba SP granitoids are consistent with an S-type classification. Investigations of S-type granites and rhyolites have flourished during the last two decades (e.g. Le Fort et al., 1987; Pichavant et al., 1988; Chappell and White, 1992; Harris and Inger, 1992; Williamson et al., 1996; Sylvester, 1998). The common point of agreement among experts of S-type felsic rocks is that they originate from the partial melting of a metasedimentary source, with or without mixing with mafic magmas.

\subsection{Petrogenesis}

The presence of two or three distinct correlation trends in some binary diagrams (e.g. $\mathrm{Mg \#}$ versus $\mathrm{CaO}$, Fig. 6) along with the parallelism between the REE and spidergram patterns (Fig. 8) suggest that the Mitwaba granitoids originated from different sources but having almost similar compositions and the petrogenetic processes involved were similar.

Several investigations (e.g. Shearer et al., 1987) have shown that fractional melting could produce geochemical trends observed in S-type leucogranites. The first liquid produced during fractional melting has the composition of minimum melt. In- 
creased melting will produce magmas characterized by higher $\mathrm{MgO}+\mathrm{FeO}+\mathrm{TiO}_{2}$ from dehydration melting of biotite at higher temperatures. Similarly, the $\mathrm{CaO}$ content of the melt will increase. The diagram $\mathrm{Mg \#}$ versus $\mathrm{CaO}$ (Fig. 6) shows distinct variation trends within both different plutons and single pluton, each of the trends originating potentially from fractional melting of a single metasedimentary source or from different source materials but with the same composition. The three distinct evolution curves in the diagram $\mathrm{Mg \#}$ versus $\mathrm{CaO}$ (Fig. 6) require at least three different metasedimentary source materials.

A critical melt fraction (>30-40 vol.\%) is required in order to separate a viscous granitic magma from its source (Wickham, 1987). Such amount of melt cannot be supplied by dehydration melting of muscovite alone because both field and experimental works showed that this reaction produces small amounts of magma (e.g. Clemens and Vielzeuf, 1987). Dehydration and breakdown of biotite is required in order to generate a large amount (up to 40\%) of melt from metasedimentary rocks (e.g. Stevens et al., 1997) to form large batholiths of S-type granites such as the Kifinga-Kisele batholith. Harris and Inger (1992) suggested that vapour-present melting of muscovite in a metapelitic source will result in peraluminous felsic melts characterized by low $\mathrm{Rb} / \mathrm{Sr}(0.7-1.6)$ versus high $\mathrm{Sr} / \mathrm{Ba}(0.5-1.6)$ ratios. Such melts should have in addition positive Eu anomalies. Vapour-absent melts from metapelites should have higher $\mathrm{Rb} / \mathrm{Sr}$ (3-6) versus lower $\mathrm{Sr} / \mathrm{Ba}(0.2-0.7)$ ratios and negative $\mathrm{Eu}$ anomalies resulting from residual plagioclase at the source. The Mitwaba granitoids have low $\mathrm{Rb} / \mathrm{Sr}$ ratios similar to those recorded in vapour-present melting of muscovite. However, their low $\mathrm{Sr} / \mathrm{Ba}$ ratios and the absence of positive $\mathrm{Eu}$ anomalies preclude the generation of these granitoids by vapour-present breakdown of muscovite. In addition, geochemical data (e.g. $\mathrm{CaO}, \mathrm{Sr}$ contents and $\mathrm{Eu} / \mathrm{Eu}^{*}$ values) suggest that these granitoids contain restitic plagioclases, supporting that this mineral was among the residues during partial melting. Therefore, the data indicate vapourabsent partial melting during the genesis of the Mitwaba peraluminous granitoids. The low $\mathrm{Rb} / \mathrm{Sr}$ values recorded in these granitoids probably indicate their derivation from a source with higher feldspar content than the Himalayan metapelites used by Harris and
Inger (1992) in their geochemical modelling of the collisional, Himalaya-type S-type granitoids.

Underplating and reaction of mafic magmas with metapelites (e.g. Patino Douce, 1995) is known to produce large volumes (up to $40 \%$ ) of SP leucogranitic melts that resemble S-type granitoids of the Lachlan Fold belt in Australia. The discrimination of S-type granitoids generated by the two distinct processes is difficult but the average chemical composition of Himalayan and Lachlan/Hercynian SP granitoids display substantial differences (e.g. Fig. 6). The Hercynian and especially the Lachlan SP granitoids show strong similarities with the Mitwaba SP granitoids (e.g. Fig. 6). Furthermore, Himalayan S-type leucogranites occur as intrusive sheets closely linked to metamorphic packages and they usually have a crustal-like isotopic signature. In contrast, the Lachlan and the Mitwaba SP granitoids occur as large plutons associated with mafic bodies and they have isotopic signatures indicating mixing between crust and mantle sources. The $\mathrm{Sr}$ isotopic ratios of Kibaran sedimentary rocks (e.g. Klerkx et al., 1987) give values close to 0.720 when recalculated back at $1381 \mathrm{Ma}$. The $\mathrm{Sr}$ isotopic compositions of granitoids from the Kibaran belt in Katanga (Cahen et al., 1984 and references therein) yield initial ${ }^{87} \mathrm{Sr} /{ }^{86} \mathrm{Sr}_{(1381 \mathrm{Ma})}$ between 0.7016 and 0.7198 , suggesting mixing between crust- and mantleoriginating materials. The diagram $\mathrm{CaO} / \mathrm{Na}_{2} \mathrm{O}$ versus $\mathrm{Al}_{2} \mathrm{O}_{3} / \mathrm{TiO}_{2}$ (Fig. 6c) suggests that Mitwaba SP granitoids could result from mixing of coeval mafic and felsic magmas exposed in the Mitwaba area (Fig. 2). To test this interpretation, we have conducted mixing calculations using: (1) the presumed mafic endmember represented by rocks with the highest Mg\# and lowest silica and alkalis from the two mafic intrusions exposed in the Mitwaba region (Lwabwe LM9 and Kidilo KM5); (2) the presumed felsic endmember corresponding to the samples with the highest silica and alkalis and the lowest $\mathrm{CaO}$ abundance (e.g. samples K2, Table 3) and chemically overlapping with the field of pelite-derived melts of Patino Douce and Johnston (1991). The quantification results in Table 4 show that, mixing 10-20\% Mitwaba mafic magmas with felsic magmas represented by the samples K2 reproduce the major elements and immobile trace elements compositions of the various petrographic groups of the Mitwaba SP granitoids $\left(\Sigma r^{2}<1\right)$. 
Table 4

Quantification of the mixing between mafic and felsic magmas to generate various Mitwaba granitoids

\begin{tabular}{|c|c|c|c|c|c|c|c|c|c|c|}
\hline \multirow{2}{*}{$\begin{array}{l}\text { Major and trace } \\
\text { elements }\end{array}$} & \multirow{2}{*}{$\begin{array}{l}\text { Fwifwi foliated } \\
\text { leucomonzogranite } \\
\text { Sample K23.5 } \\
\text { A }\end{array}$} & \multicolumn{2}{|c|}{ Mixed magmas ${ }^{\mathrm{a}}$} & \multirow{2}{*}{$\begin{array}{l}\text { Calcul-ated } \\
\text { mixture }^{\mathrm{a}}\end{array}$} & $\begin{array}{l}\text { Square } \\
\text { difference }^{\mathrm{a}}\end{array}$ & $\begin{array}{l}\text { Kabonvia } \\
\text { Granodioritic } \\
\text { augen gneiss }{ }^{b}\end{array}$ & \multicolumn{2}{|c|}{ Mixed magmas ${ }^{b}$} & \multirow{2}{*}{$\begin{array}{l}\text { Calculated } \\
\text { mixture }\end{array}$} & $\begin{array}{l}\text { Square } \\
\text { difference }^{b}\end{array}$ \\
\hline & & $\begin{array}{l}\text { Felsic }(\%) \\
81.0\end{array}$ & $\begin{array}{l}\text { Mafic (\%) } \\
19.0\end{array}$ & & $(A-B)^{2}$ & $\begin{array}{l}\text { Sample K12.1 } \\
\text { A }\end{array}$ & $\begin{array}{l}\text { Felsic }(\%) \\
81.0\end{array}$ & $\begin{array}{l}\text { Mafic (\%) } \\
19.0\end{array}$ & & $(A-B)^{2}$ \\
\hline $\mathrm{SiO}_{2}(\%)$ & 71.81 & 60.49 & 10.52 & 71.01 & 0.64 & 69.39 & 60.49 & 9.14 & 69.63 & 0.06 \\
\hline $\mathrm{TiO}_{2}$ & 0.42 & 0.14 & 0.15 & 0.28 & 0.02 & 0.51 & 0.14 & 0.13 & 0.26 & 0.06 \\
\hline $\mathrm{Al}_{2} \mathrm{O}_{3}$ & 13.22 & 10.40 & 2.69 & 13.09 & 0.02 & 13.79 & 10.40 & 3.14 & 13.54 & 0.06 \\
\hline $\mathrm{FeO}_{t}$ & 2.07 & 1.00 & 1.39 & 2.38 & 0.10 & 3.47 & 1.00 & 2.38 & 3.37 & 0.01 \\
\hline $\mathrm{MnO}$ & 0.03 & 0.02 & 0.04 & 0.06 & 0.00 & 0.07 & 0.02 & 0.02 & 0.04 & 0.00 \\
\hline $\mathrm{MgO}$ & 0.86 & 0.41 & 0.82 & 1.24 & 0.14 & 1.66 & 0.41 & 1.37 & 1.78 & 0.02 \\
\hline $\mathrm{CaO}$ & 1.83 & 0.55 & 1.10 & 1.65 & 0.03 & 1.36 & 0.55 & 1.01 & 1.56 & 0.04 \\
\hline $\mathrm{Na}_{2} \mathrm{O}$ & 3.12 & 3.13 & 0.14 & 3.28 & 0.03 & 2.68 & 3.13 & 0.02 & 3.15 & 0.22 \\
\hline \multirow[t]{2}{*}{$\mathrm{K}_{2} \mathrm{O}$} & 3.75 & 3.09 & 0.51 & 3.61 & 0.02 & 4.71 & 3.09 & 0.91 & 4.00 & 0.50 \\
\hline & & & & & $\sum r^{2}=0.99$ & & & & & $\sum r^{2}=0.97$ \\
\hline $\mathrm{La}(\mathrm{ppm})$ & 49.50 & 46.58 & 2.49 & 49.06 & 0.19 & 50 & 46.58 & 3.82 & 50.39 & 0.15 \\
\hline $\mathrm{Hf}$ & 5.00 & 4.86 & 0.19 & 5.05 & 0.00 & 5 & 4.86 & 0.19 & 5.05 & 0.00 \\
\hline $\mathrm{Sm}$ & 7.70 & 7.13 & 0.57 & 7.70 & 0.00 & 7.5 & 7.13 & 0.57 & 7.70 & 0.04 \\
\hline $\mathrm{Nd}$ & 41.00 & 36.05 & 5.19 & 41.23 & 0.05 & 41 & 36.05 & 5.00 & 41.05 & 0.00 \\
\hline $\operatorname{Pr}$ & 11.30 & 10.13 & 0.72 & 10.85 & 0.21 & 11 & 10.13 & 0.57 & 10.70 & 0.09 \\
\hline $\mathrm{Lu}$ & 0.40 & 0.81 & 0.04 & 0.85 & 0.20 & 0.4 & 0.81 & 0.04 & 0.85 & 0.20 \\
\hline $\mathrm{Eu}$ & 1.40 & 0.89 & 0.15 & 1.04 & 0.13 & 1.4 & 0.89 & 0.15 & 1.04 & 0.13 \\
\hline $\mathrm{Tm}$ & 0.50 & 0.73 & 0.06 & 0.79 & 0.08 & 0.4 & 0.73 & 0.06 & 0.79 & 0.15 \\
\hline \multirow[t]{2}{*}{$\mathrm{Tb}$} & 1.00 & 1.22 & 0.08 & 1.29 & 0.08 & 1 & 1.22 & 0.11 & 1.33 & 0.11 \\
\hline & & & & & $\sum r^{2}=0.95$ & & & & & $\sum r^{2}=0.88$ \\
\hline
\end{tabular}

a Parent magmas used to generate the Fwifwi leucomonzogranite are: Kisele monzogranite (sample K2.3, Table 3). and the Kidilo mafic intrusive (KM) having the following chemical composition (\%): $\mathrm{SiO}_{2}: 55.38 ; \mathrm{TiO}_{2}: 0.77 ; \mathrm{Al}_{2} \mathrm{O}_{3}: 14.17 ; \mathrm{Fe}_{2} \mathrm{O}_{3}: 7.3 ; \mathrm{MnO}: 0.22 ; \mathrm{MgO}: 4.3 ; \mathrm{CaO}: 5.81 ; \mathrm{Na}_{2} \mathrm{O}: 0.76$; and $\mathrm{K} 2 \mathrm{O}: 2.7$. Traces (ppm): La: 13.1; $\mathrm{Hf}$ : 1; Sm: 3; Nd: 27.3; Pr: 3.8; Lu: 0.2; Eu: 0.8; Tm: 0.3; and Tb: 0.4.

b Parent magmas used to generate the Kabonvia granodiorite are: the Kisele monzogranite (sample K2.3, Table 3) and Lwabwe mafic intrusive (LM) having the following chemical composition (\%): $\mathrm{SiO}_{2}: 48.10 ; \mathrm{TiO}_{2}: 0.66 ; \mathrm{Al}_{2} \mathrm{O}_{3}: 16.50 ; \mathrm{Fe}_{2} \mathrm{O}_{3}: 12.50 ; \mathrm{MnO}: 0.10 ; \mathrm{MgO}: 7.21 ; \mathrm{CaO}: 5.32 ; \mathrm{Na}_{2} \mathrm{O}: 0.10$; and $\mathrm{K}_{2} \mathrm{O}: 4.79$. Traces (ppm): La: 20.09; Hf: 1; Sm: 3; Nd: 26.3; Pr: 3; Lu: 0.2; Eu: 0.8; Tm: 0.3; and Tb: 0.6. (Mafic rocks data from Kokonyangi et al., unpublished.) 


\section{Geotectonic implications}

Three geotectonic settings will be evaluated for the Kibaran granitoids from the Mitwaba area and for coeval and petrologically similar Kibaran granitoids exposed in Burundi, Rwanda and NE Tanzania.

The first model is an extensional (plate divergent) setting, i.e. a continental rift setting proposed by Klerkx et al. (1987). In modern tectonic environments, nowhere very large S-type granitic batholiths unrelated to felsic A-type intrusions have been reported in plate divergent setting. We have shown that the Mitwaba granitoids were emplaced during a compressional $\left(D_{1}\right)$ tectonic event, which preclude a pristine extensional environment. Plate divergent settings are marked by extensional tectonic structures and alkaline-peralkaline felsic rocks with a very specific high-field-strength geochemical signature unknown in the Kibaran granitoids.

The second model is a continental collision setting similar to the Himalaya where S-type granitoids are widespread (e.g. Le Fort et al., 1987). In such setting, S-type leucogranites mark an advanced stage of crustal thickening by stacking of thrust sheets. In the Mitwaba area, the S-type batholiths were emplaced $\sim 300 \mathrm{Ma}$ before $\mathrm{D}_{2}$, the climax of collisional deformation tentatively dated at $\sim 1080 \mathrm{Ma}$ in this paper. $\mathrm{D}_{1}$ deformation event does not seem to represent an advanced stage of crustal thickening in the Kibaran belt. Himalayantype S-type granitoids usually plot well inside the collisional granites field in Pearce (1996) discrimination diagram. The syn- $\mathrm{D}_{1}$ Mitwaba granitoids originate also from partial melting of sedimentary rocks but in contrast to the Himalayan S-type granitoids, they plot at the boundary between volcanic-arc, collisional and within-plate granite fields (Fig. 7). We infer that this peculiar feature result from the mixing between mafic and felsic magmas during the genesis of the Mitwaba SP granitoids. Huppert and Sparks (1988) stressed the important role of mafic magmas in the generation of granitoids in the crust and experimental work (e.g. Patino Douce, 1995) showed that a large amount (up to 40 vol.\%) of felsic melts $\left(>70 \% \mathrm{SiO}_{2}\right)$ could be produced by the interaction of hot mafic magma with metapelitic material. The degree of crustal melting required for generating, during the early stage of an orogen, the large S-type granitic batholiths known in the Kibaran belt could not be achieved without input of ex- ternal heat. Although part of heat required to generate the large S-type granitic batholiths is probably from radioactive decay as suggested by high- $U$ contents in the restitic zircons (see Table 2), we are suggesting that the $\sim 1.38 \mathrm{Ga}$ mafic rocks closely associated to the Kibaran SP granitoids (e.g. Fig. 2) were probably the major source of heat required for melting the Kibaran metasedimentary rocks of the Mitwaba group. The Mulumbi group metasedimentary rocks post-date the emplacement of the syn- $\mathrm{D}_{1}$ Kibaran SP granitoids and thus cannot be considered as their potential source.

A mechanism that can supply heat for crustal melting is underplating of mafic magma at the Moho and/or within the crustal metasedimentary package. This process is important both in volcanic-arc (e.g. Hyndman and Foster, 1988) and in extensional setting (e.g. Bergantz, 1989). Structural investigations indicated that the Mitwaba area was undergoing compressional tectonics $\left(\mathrm{D}_{1}\right)$ during the emplacement of the S-type granitoids and thus cannot mark an extensional regime. Therefore, the Mitwaba S-type granitoids formed in a continental-arc setting as previously suggested by Kampunzu et al. (1986). Geochemical data related to mafic magmas emplaced at the same time as the SP granitoids also record a continental arc setting (Kokonyangi, unpublished data). The two cratons involved during this convergence are the Congo craton to the northwest and the Bangweulu block/Tanzania craton to the southeast. The absence of any possibility of correlation of geological units on the two sides of the Kibaran belt indicates that the two-cratonic blocks were exotic to each other. The formation of S-type felsic melts in subduction zones is a well-known fact in modern orogens (e.g. Pichavant et al., 1988). The arc signature documented in the mafic magmas emplaced at the same time as the SP granitoid melts in the Mitwaba batholiths supports this interpretation. The petrogenesis and geodynamic setting during the emplacement of Kibaran peraluminous granitoids present several analogies with SP granitoids in the Lachlan Fold belt and in the Variscan belt in Europe (e.g. Bea et al., 1999). The Variscan peraluminous granitoids were produced in a belt thickened by underthrusting of the lower crust (Azor et al., 1994), i.e. a crust thickened by subduction rather than by continental collision. Bea et al. (1999) emphasized the production of Variscan SP granitoids during an orogeny-related extensional event separat- 
ing two compressional events. In this study, we do not see evidence of orogeny-related extensional tectonics during the emplacement of the Kibaran granitoids. Arc mafic magmas are almost coeval with SP crustal felsic melts and therefore we infer that this is an important parameter for the genesis of peraluminous granitoids in these belts. However, Bea et al. (1999) suggested that the amount of heat advected to the middle crust by mafic magmas was insufficient to cause significant melting of crustal rocks and preferred intra-crustal radioactive decay as the main heat source. An important observation that can be made from the data of Bea et al. (1999) is that the "mafic precursor" rocks in the Variscan belt show abnormal compositions indicating that they are hybrid rocks from various degree of mixing of mantle- and crustoriginating melts. Such feature imply that a large proportion of this mixture could be trapped below the currently exposed crustal level because, in such a scenario, the most viscous felsic melt will impend the ascent to shallow level of the less viscous mafic melts. Whatever the model adopted, what is critical is that the Mitwaba S-type granitoids were formed and emplaced within a plate convergent setting, most probably during a subduction event.

\section{Conclusion}

New, high precision ages presented in this paper show that the emplacement of the three generations $\left(G_{1}, G_{2}\right.$ and $\left.G_{3}\right)$ of granitoids previously reported to span the period $1370 \pm 25 \mathrm{Ma}$ to $1094 \pm 50 \mathrm{Ma}$ in the Kibaran belt occurred within a short period of time at $1381 \pm 8 \mathrm{Ma}$ and date the first deformation event $\mathrm{D}_{1}$ in the Kibaran orogenic system in central Africa. The Kisele monzogranite gneiss was strongly deformed and metamorphosed during $\mathrm{D}_{2}$ and hosts metamorphic zircons yielding an age of $1079 \pm 14 \mathrm{Ma}$, which sets the age of the $\mathrm{D}_{2}$ deformation event and related metamorphism in the Mitwaba area.

The major- and trace-element compositions (e.g. high $\mathrm{SiO}_{2}, \mathrm{Al}_{2} \mathrm{O}_{3}$, ASI ratios, normative corundum, low $\mathrm{CaO}$, Th and $\mathrm{U}$ contents and high $\mathrm{Rb} / \mathrm{Zr}$ and $\mathrm{Rb} / \mathrm{Th}$ ) of the syn-kinematic $\mathrm{D}_{1}$ Kibaran granitoids from the Kibaran belt are consistent with their genesis by partial melting of metasedimentary rocks of the Mitwaba group. The emplacement of mafic and felsic igneous rocks in the Mitwaba occurred within the same period of time were coeval. The source of heat for the production of the large S-type granitic batholiths in the Kibaran is inferred to be underplating of arc mafic magmas at the base and within the crust. However, additional heat could have been supplied by radioactive decay from high $\mathrm{U}$, Th and $\mathrm{K}$ metasedimentary rocks as shown by high U-content of restitic zircon and high K-content of these granitoids. Available data do not support a Himalaya-type continental collision setting during the genesis of these S-type granitoids. A model involving subduction, genesis of arc mafic magmas, substantial intra-crustal melting of metasedimentary rocks triggered by the emplacement of arc mafic magmas and mixing in variable proportions of these mafic and felsic magmas is taken to be the dominant factor during the genesis of these peraluminous granitoids.

\section{Acknowledgements}

J.K. acknowledges the Japanese Ministry of Education (MONBUKAGAKUSHO) for the scholarship. We acknowledge the reviews by R. Van Schmus and J.-P. Liegeois. This is a contribution to the Japanese Society for Promotion of Science (Research Project No. 1337005, Leader M. Arima) and the IGCP 368 and 440. J.K. acknowledges M. Bwanambao, T. Pierrot, K. Furuyama, I. Reiko and S. Biju for various contributions to this work.

\section{References}

Abdel-Rahman, A.M., 1994. Nature of biotite from alkaline, calcalkaline and peraluminous magmas. J. Petrol. 35, 525-541.

Altherr, R., Holl, A., Hegner, E., Langer, C., Kreuzer, H., 2000. High-potassium, calc-alkaline I-type plutonism in the European Variscides: northern Vosges (France) and northern Schwarzwald (Germany). Lithos 50, 51-73.

Azor, A., González Lodeiro, F., Simancas, F., 1994. Tectonic evolution of the boundary between the central Iberian and OssaMorena zones (Variscan belt, southwest Spain). Tectonics 13, 45-61.

Barbarin, B., 1999. A review of relationships between granitoids types, their origins and their geodynamic environments. Lithos 46, 605-626.

Batchelor, R.A., Bowden, P., 1985. Petrogenetic interpretation of granitoid rock series using multicationic parameters. Chem. Geol. 48, 43-55. 
Bea, F., Montero, P., Molina, J.F., 1999. Mafic precursors, peraluminous granitoids, and late lamprophyres in the Avila batholith: a model for the generation of Variscan batholiths in Iberia. J. Geol. 107, 399-419.

Bergantz, G.W., 1989. Underplating and partial melting: implication for the melt generation and extraction. Science 245 , 1093-1095.

Boutakoff, N., 1939. Géologie des terrains situés à l'Ouest et au Nord du fossé téctonique du Kivu. Mém. Inst. Géol. Univ. Louvain 9, 7-207.

Cahen, L., 1954. Géologie du Congo Belge. Vaillant-Carmanne, Liège, Belgium, $580 \mathrm{pp}$.

Cahen, L., Delhal, J., Vail, J.R., Bonhomme, M., Ledent, D., 1984. The Geochronology and Evolution of Equatorial Africa. Clarendon Press, Oxford, 496 pp.

Chappell, B.W., White, J.A.R., 1992. I- and S-type granites in the Lachlan Fold Belt. Trans. R. Soc. Edinb. Earth Sci. 83, 1-26.

Clemens, J.D., Vielzeuf, D., 1987. Constraints on melting and magma production in the crust. Earth Planet. Sci. Lett. 86, 287-306.

Compston, W., Williams, I.S., Kirschvink, J.L., Zhang, Z., Ma, G., 1992. Zircon U-Pb ages for the Early Cambrian time-scale. J. Geol. Soc. Lond. 149, 171-184.

Cumming, G.L., Richards, J.R., 1975. Ore lead isotope ratios in a continuously changing Earth. Earth Planet. Sci. Lett. 28, 155171.

De La Roche, H., Leterrier, J., Grande Claude, P., Marchal, M., 1980. A classification of volcanic and plutonic rocks using R1R2 diagrams and major element analyses-its relationship and current nomenclature. Chem. Geol. 29, 183-210.

Didier, J., 1973. Granites and Their Enclaves: The Bearing of Enclaves on the Origin of Granites. Elsevier Publishing Co., Amsterdam, 393 pp.

Eberhardt, P., Geiss, J., Von Gunten, H., Houtermans, F.G., Signer, P., 1956. Mésure à l'âge de l'yttrocrasite de Mitwaba (Katanga) par la méthode de plomb. II. Mesures isotopiques. Bull. Soc. Belg. Géol. LXV, 251-255.

Fernandez-Alonso, M., Lavreau, J., Klerkx, J., 1986. Geochemistry and geochronology of the Kibaran granites in Burundi, central Africa: implication for the Kibaran orogeny. Chem. Geol. 57, 217-234.

Harris, N.B.W., Inger, S., 1992. Trace element modelling of pelitederived granites. Contrib. Miner. Petrol. 110, 46-56.

Harris, N.B.W, Pearce, J.A., Tindle, A.G., 1986. Geochemical characteristics of collision-zone magmatism. In: Coward, M.P., Ries, A.C. (Eds.), Collision Tectonics, vol. 19. Spec. Publ. Geol. Soc. Lond., pp. 67-81.

Huppert, H.E., Sparks, R.S.J., 1988. The generation of granitic magma by intrusion of basalt into continental crust. J. Petrol. 29, 599-624.

Hyndman, D.W., Foster, D.A., 1988. The role of tonalites and mafic dykes in the generation of Idaho batholith. J. Petrol. 25, 894-929.

Ikingura, J.R., Reynolds, P.H., Watkinson, D.H., Bell, K., 1992. ${ }^{40} \mathrm{Ar} /{ }^{39} \mathrm{Ar}$ dating of micas from granites of NE Kibaran belt (Karagwe-Ankolean). NW Tanzania J. Afr. Earth Sci. 15, 501511.
Ishihara, S., 1977. The magnetite-series and ilmenite-series granitic rocks. Min. Geol. 27, 293-305.

Kampunzu, A.B., 2001. Assembly and break-up of Rodinia-no link with Gondwana assembly. Gondwana Res. 4, 647-650.

Kampunzu, A.B., Rumvegeri, B.T., Kapenda, D., Lubala, R.T., Caron, J.P., 1986. Les Kibaran d'Afrique centrale et orientale: une chaîne de collision. UNESCO, Geol. Econ. Dev., Newslett. 5, 125-137.

Kampunzu, A.B., Akanyang, P., Mapeo, R.B.M., Modie, B.N., Wendorff, M., 1998. Geochemistry and tectonic significance of Mesoproterozoic Kgwebe metavolcanic rocks in northwest Botswana: implications for the evolution of the Kibaran Namaqua-Natal Belt. Geol. Mag. 133, 669-683.

Kapenda, D., Kampunzu, A., Cabanis, B., Namegabe, B., Tshimanga, M., 1998. Petrology and geochemistry of postkinematic mafic rocks from the Paleoproterozoic Ubendian belt, NE Katanga (Democratic Republic of Congo). Geol. Rundsch. 87, 345-362.

Klerkx, J., Liégeois, J.P., Lavreau, J., Claessen, W., 1987. Crustal evolution of northern Kibaran belt in eastern and central Africa. In: Kröner, A. (Ed.), Proterozoic Lithospheric Evolution, vol. 17. Am. Geophys. Union, Geodyn. Ser., pp. 217-233.

Kokonyangi, J., Okudaira, T., Kampunzu, A.B., Yoshida, M., 2001. Geological evolution of the Kibaran belt, Mitwaba, Democratic Republic of Congo, Central Africa. Gondwana Res. 4, 663-664.

Ledent, 1979. Données géochronologiques relatives aux granites Kibariens de type A ou G1 et B ou G2 du Shaba, du Rwanda, du Burundi et du Sud-Ouest de 1'Ouganda. Mus. Roy. Afr. Centr., Tervuren, Dépt. Géol. Min., Rapp. Ann. 1978. pp. 101-105.

Le Fort, P., Cuney, M., Deniel, C., France-Lanord, C., Sheppard, S.M.F., Upreti, B.N., Vidal, Ph., 1987. Crustal generation of Himalayan leucogranites. Tectonophysics 134, 39-57.

Ludwig, K.R., 1998. On the treatment of concordant uranium-lead ages. Geochim. Cosmochim. Acta 62, 665-676.

Ludwig, K.R., 2000. SQUID 1.00, A User's Manual. Berkeley Geochronology Center Spec. Publ., vol. 2. Berkeley, CA, 17 pp.

Mezger, K., Krogstad, E.J., 1997. Interpretation of discordant U-Pb zircon ages: an evaluation. J. Met. Petrol. 15, 127-140.

Miller, C.F., 1985. Are strongly peraluminous magmas derived from pelitic sedimentary sources? J. Geol. 93, 673-689.

Peaces, J.B., Miller, J.D., 1989. Precise U-Pb ages of Duluth Complex and related mafic intrusions, Northeastern Minnesota: geochronological insights to physical, petrogenic, paleomagnetic and tectonomagmatic processes associated with the 1.1 Ga Midcontinent rift system. J. Geophys. Res. 98, 13997-14013.

Patino Douce, A.E., 1995. Experimental generation of hybrid silicic melts by reaction of high-Al basalt with metamorphic rocks. J. Geophys. Res. 100, 15623-15639.

Patino Douce, A.E., Johnston, A.D., 1991. Phase equilibria and melt productivity in pelitic system: implication for the origin of peraluminous granitoids and aluminous granulites. Contrib. Miner. Petrol. 107, 202-218.

Pearce, J.A., 1996. Sources and settings of granitic rocks. Episodes 19, 120-125.

Pearce, J.A., Harris, N.B.W., Tindle, A.G., 1984. Trace elements discrimination diagrams for the tectonic interpretation of granitic rocks. J. Petrol. 25, 956-983. 
Pichavant, M., Kontak, D.J., Herrera, J.V., Clark, A.H., 1988. The Miocene-Pliocene Macusani volcanics. SE Perou. I. Mineralogy and magmatic evolution of a two-micas alumino-silicate-bearing ignimbrite suite. Contrib. Miner. Petrol. 100, 300-324.

Roddick, J.C., Van Breemen, O., 1994. U-Pb zircon dating: a comparison of ion microprobe and single grain conventional analyses. Radiogenic Age and Isotopic Studies: Report 8. Geological Survey of Canada, Current Research 1994-F, pp. 1-9.

Romer, R.L., Lehmann, B., 1995. U-Pb columbite age of Neoproterozoic Ta-Nb mineralization in Burundi. Econ. Geol. 90, 2303-2309.

Rumvegeri, B.T., 1991. Tectonic significance of Kibaran structures in central and eastern Africa. J. Afr. Earth Sci. 13, 267-276.

Scaillet, B., France-Lanord, C., Le Fort, P., 1990. BadrinathGangotri plutons (Garhwal India): petrological and geochemical evidence for fractionation processes in a high Himalayan leucogranite. J. Volcan. Geotherm. Res. 44, 163-188.

Saunders, A.D., Norry, M.J., Tarney, J., 1991. Fluid influences on the trace element composition of subduction zone magmas. Philos. Trans. R. Soc. Lond. A335, 377-392.

Shearer, C.K., Papike, J.J., Laul, J.C., 1987. Mineralogical and chemical evolution of a rare-element granite-pegmatite system: Harney Peak granite, Black Hills. South Dakota. Geochim. Cosmochim. Acta 51, 473-486.

Speer, J.A., 1984. Micas in igneous rocks. Miner. Soc. Am. Rev. Min. 13, 229-356.

Stevens, G., Clemens, J.D., Droop, G.T.R., 1997. Melt production during granulite-facies anatexis: experimental data from "primitive" metasedimetary protolith. Contrib. Miner. Petrol. 128, 352-370.

Streckeisen, A.L., 1976. To each plutonic rock its proper name. Earth Sci. Rev. 12, 1-33.

Sun, S., McDonough, W.F., 1989. Chemical and isotopic systematics of ocean basalts: implications for mantle composition and processes. In: Saunders, A.D., Norry, M.J. (Eds.), Magmatism in the Ocean Basins, vol. 42. Geol. Soc. Lond. Spec. Publ., pp. 313-345.

Sylvester, P.J., 1998. Post-collisional strongly peraluminous granites. Lithos 45, 29-44.

Tack, L., Liégeois, J.-P., Deblond, A., Duchesne, J.C., 1994. Kibaran A-type granitoids and mafic rocks generated by two mantle sources in a late orogenic setting (Burundi). Precambrian Res. 68, 323-356.

Tarney, J., Jones, C.E., 1994. Trace element geochemistry of orogenic igneous rocks and crustal growth models. J. Geol. Soc. Lond. 151, 855-868.

Taylor, S.R., McLennan, S.M., 1985. The Continental Crust: Its Composition and Evolution. Blackwell, Oxford, 312 pp.

Watson, E.B., Harrison, T.M., 1983. Zircon saturation revisited: temperature and composition affecting variety of crustal magma types. Earth Planet. Sci. Lett. 64, 295-304.

Wickham, S.M., 1987. The generation and emplacement of granitic magmas. J. Geol. Soc. Lond. 144, 281-297.

Williams, I.S., 2001. Response of detrital zircon and monazite, and their $\mathrm{U}-\mathrm{Pb}$ isotopic systems, to regional metamorphism and host-rock partial melting, Cooma Complex, southestern Australia. Aust. J. Earth Sci. 48, 557-580.

Williams, I.S., Claesson, S., 1987. Isotopic evidence for the Precambrian provenance and Caledonian metamorphism of high grade paragneisses from the Seve Nappes Scandinavian Caledonides. II. Ion microprobe zircon U-Th-Pb. Contrib. Miner. Petrol. 97, 205-217.

Williamson, B.J., Shaw, A., Downes, H., Thirlwall, M.F., 1996. Geochemical constraints on the genesis of Hercynian two micas leucogranites from the Massif Central. France Chem. Geol. 127, 25-42.

Winkler, H.G.F., Boese, M., Marcopoulos, T., 1975. Low temperature granitic melts. Neues Jahrb. Miner. Monatsh. $245-268$. 